\title{
On certain cohomological operations.
}

\author{
By Tsuneyo Yamanoshita
}

(Received June 12, 1956)

\section{Introduction}

Let $A, B$ be given abelian groups and $m, n$ fixed non-negative integers. Then Serre [7] has defined as follows the cohomology operation relative to $(A, B, m, n)$. It is a mapping $C$ defined for each $C W$-complex $K$ of the $m$-th cohomology group $H^{m}(K, A)$ into $H^{n}(K, B)$, such that the following diagram is commutative

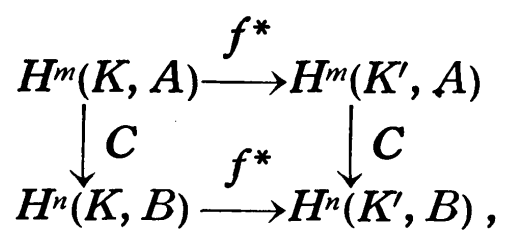

where $K^{\prime}$ is another $C W$-complex, $f^{*}$ the homomorphism of the cohomology group of $K$ into that of $K^{\prime}$ induced by a simplicial mapping $f: K^{\prime} \rightarrow K$. In generalizing this notion, we shall now consider operations of the following kind. Our mapping $C$ has as its domain of definition a subgroup $S$ of $H^{m}(K, A)$ and as its range a factor group $H^{n}(K, B) / M$ of $H^{n}(K, B)$. Once $C$ is given, an subgroup $S=S(K)$ of $H^{m}(K, A)$ and the subgroup $M=M(K)$ of $H^{n}(K, B)$ are thus defined by $K$; we postulate now

$$
\begin{aligned}
& S\left(K^{\prime}\right) \subset f^{*}(S(K)), \\
& M\left(K^{\prime}\right) \subset f^{*}(M(K))
\end{aligned}
$$

for every simplicial mapping $f: K^{\prime} \rightarrow K$. $C$ will be then called cohomological operation if the following diagram is commutative

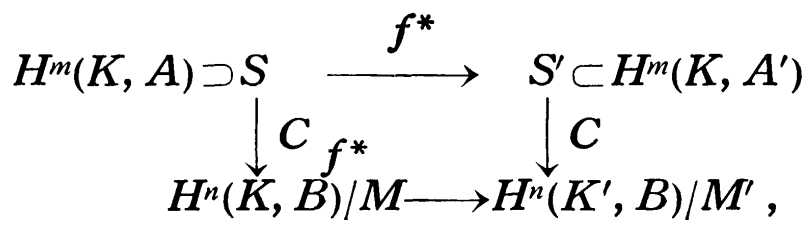


whence $S^{\prime}=S\left(K^{\prime}\right), M^{\prime}=M\left(K^{\prime}\right)$. For example, the Adem operation $\Phi$ introduced in [1] is a cohomological operation in our sense.

More generally, $X$ being any topological space, and the cohomology group $H^{*}(X, G)$ of $X$ with an abelian group $G$ as coefficient being the singular one, we can correspondingly define the cohomological operation in an obvious manner.

$Z$ means as usual the additive group of integers; $Z_{n}$ the group $Z$ $\bmod n ; p$ a prime number. The meanings of $F_{h}, G_{h}, f_{h}, g_{h}, f_{h}^{\prime}, g_{h}^{\prime}$ will be clear from the following exact sequences

$$
\begin{aligned}
& 0 \longrightarrow Z \stackrel{F_{h}}{\longrightarrow} Z \stackrel{G_{h}}{\longrightarrow} Z_{p^{h}} \longrightarrow 0, \\
& 0 \longrightarrow Z_{p^{h}} \stackrel{f_{h}}{\longrightarrow} Z_{p^{h+1}} \stackrel{g_{h}}{\longrightarrow} Z_{p} \longrightarrow 0, \\
& 0 \longrightarrow Z_{p} \stackrel{f_{h}^{\prime}}{\longrightarrow} Z_{p^{h+1}} \stackrel{g_{h}^{\prime}}{\longrightarrow} Z_{p^{h}} \longrightarrow 0 .
\end{aligned}
$$

The coboundary operators associated with $\left(\mathrm{I}_{h}\right),\left(\mathrm{II}_{h}\right),\left(\mathrm{III}_{h}\right)$ (cf. $\left.\S 1\right)$ are denoted by $1 / p^{h} \delta, \delta_{h}, \delta_{h}^{\prime}$ respectively.

$\S 1$ contains algebraic preliminaries and topological meanings of Bockstein operators (cf. $\S 1$ Theorem 2.1.) and in particular of $1 / p^{h} \delta$, $\delta_{h}, \delta_{h}^{\prime}$.

In $\S 2$, we define the operations $\Delta_{p}^{i}, i=1, \cdots$ and give their fundamental properties. $\Delta_{p}^{1}$ is nothing other than $\delta_{1}=\delta_{1}^{\prime} ; \Delta_{p}^{2}$ maps $\operatorname{Ker} \Delta_{p}^{1} \cap H^{n}\left(X, Z_{p}\right)$ into $H^{n+1}\left(X, Z_{p}\right) / \operatorname{Im} \delta_{1}^{\prime}$ homomorphically ; in general, $\Delta_{p}^{i}, i \geqq 1$, maps $\operatorname{Ker} \Delta_{p}^{i-1} \cap H^{n}\left(X, Z_{p}\right)$ into $H^{n+1}\left(X, Z_{p}\right) / \operatorname{Im} \delta_{h-1}^{\prime}$ homomorphically. The knowledge of the effect of $\Delta_{p}^{i}, i=1,2, \cdots$ will suffice to determine the $p$-primary component of $H^{*}(X, Z)$. (Theorem 1.1 in $\S 2$ ).

Furthermore, if $E \supset F$, the knowledge on the effects of $\Delta_{p}^{i}$ on $H^{*}\left(E, F ; Z_{p}\right)$ and on $H^{*}\left(E, Z_{p}\right)$ will give us some information on the effect of $\Delta_{p}^{i}$ on $H^{*}\left(F, Z_{p}\right)$. These circumstances, useful in the computation of homotopy groups of spheres and $C W$-complexes, are expounded in $\S 3$. Professor $H$. Toda has kindly communicated to me, that Professor H. Cartan and himself have also obtained the same results as our Theorems $3.2,3.5,3.7$ and utilized them to compute the stable homotopy groups of spheres.

In $\S 4$, we define the operations $P_{p}^{h}, 1 / p P_{p}^{h}$ and allied operations. $P_{2}^{1}$ is nothing but the Pontrjagin square $[11,15] \cdot 1 /{ }_{p} P_{p}^{1}$ is useful in 
giving the generators of $H^{*}\left(\Omega\left(S^{n}\right), Z_{p}\right)$, where $\Omega\left(S^{n}\right)$ is the loop space of the $n$-sphere $S^{n}$.

The author intends to publish in a forthcoming paper the applications of the results of this paper to the homotopy theory. He wishes to express his hearty thanks to his friends $N$. Yoneda, $Y$. Saito, T. Nakamura, A. Hattori, who have given him valuable suggestions through kind criticisms and discussions and also to Professor S. Iyanaga for his constant encouragement during the preparation of this paper.

\section{§ 1. Algebraic preliminaries}

1. Torsion products and extension groups [14]. Let $A, B$ be (abelian) groups. If we represent $A$ as a factor group of a free (abelian) group $F$, then the kernel $R$ of the epimorphism $F \rightarrow A$ is also free. Let $T$ be the kernel of the homomorphism $R \otimes B \rightarrow F \otimes B$ and $H$ the cokernel of the homomorphism $\operatorname{Hom}(F, B) \rightarrow \operatorname{Hom}(R, B)$. These are invariants of the pair $(A, B)$, and are denoted respectively by $A * B$ (torsion product of $A$ and $B$ ) and $\operatorname{Ext}(A, B)$ (extension group of $B$ by $A) . \quad A * B$ is a covariant functor in $A$ and in $B$. $\operatorname{Ext}(A, B)$ is a contravariant functor in $A$ and a covariant functor in $B$. We list here some properties of torsion products and extension groups.

1.1. $A * B \approx B * A$.

1.2. Let

$$
0 \longrightarrow A \longrightarrow B \longrightarrow C \longrightarrow 0
$$

be an exact sequence and $G$ a group. Then we have an exact sequence

$$
0 \longrightarrow A * G \longrightarrow B G \longrightarrow C * G \longrightarrow A \otimes G \longrightarrow B \otimes G \longrightarrow C \otimes G \longrightarrow 0 \text {. }
$$
sum.

1.3. The functor $A * B$ commutes with the formation of direct 1.4. We have

$$
Z * A=0 \text { and } Z_{n} * A={ }_{n} A,
$$

where ${ }_{n} A$ is the subgroup of $A$ consisting of a with $n a=0$.

1.5. Under the same hypothesis as in 1.2, we have the following exact sequences : 
$0 \longleftarrow \operatorname{Ext}(A, G) \longleftarrow \operatorname{Ext}(B, G) \longleftarrow \operatorname{Ext}(C, G) \longleftarrow \operatorname{Hom}(A, G) \longleftarrow$

$\operatorname{Hom}(B, G) \longleftarrow \operatorname{Hom}(C, G) \longleftarrow 0$,

$0 \longrightarrow \operatorname{Hom}(G, A) \longrightarrow \operatorname{Hom}(G, B) \longrightarrow \operatorname{Hom}(G, C) \longrightarrow \operatorname{Ext}(G, A) \longrightarrow$

$\operatorname{Ext}(G, B) \longrightarrow \operatorname{Ext}(G, C) \longrightarrow 0$.

1.6. The functor $\operatorname{Ext}(A, B)$ commutes with the formation of direct sum (finite).

1.7. We have

$$
\operatorname{Ext}(Z, A)=0 \text { and } \operatorname{Ext}\left(Z_{n}, A\right) \approx A / n A,
$$

where $n A$ is the subgroup of $A$ consisting of the elements $n a, a \in A$.

2. Coboundary operators associated with exact sequences of coefficients. When we have an exact sequence $(S)$, we have clearly the following exact sequence of singular cohomology groups of a space $X$ :

$$
\cdots \longrightarrow H^{n}(X, A) \longrightarrow H^{n}(X, B) \longrightarrow H^{n}(X, C) \stackrel{\delta}{\longrightarrow} H^{n+1}(X, A) \longrightarrow \cdots,
$$

whence $\delta$ is the coboundary operator associated with $(S) . \quad \delta$ is defined in the following way. We have the following commutative diagram for cochain groups:

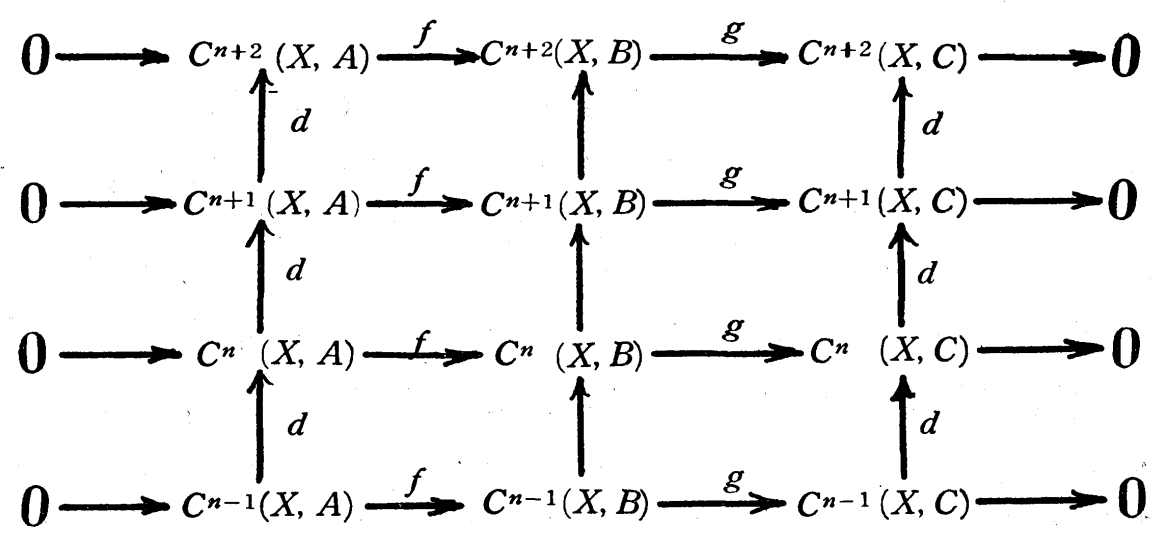

Diagram 1.

Let $c$ be a representative cocycle of class $\{c\} \in H^{n}(X, C)$. From the above diagram, it is easily seen $\left\{f^{-1} \circ d \circ g^{-1}(c)\right\}$ is uniquely determined for the class $\{c\}$ as an element of $H^{n+1}(X, A)$. We denote this by $\delta\{c\}$. Obviously $\delta: H^{n}(X, C) \rightarrow H^{n+1}(X, A)$ is a homomorphism. 
We shall determine the kernel and the image of $\delta$. Denote by $L_{n}$ the $n$-th homology group $H_{n}(X, Z)$. Then, as is well-known, we have

$$
H^{n}(X, G) \approx \operatorname{Ext}\left(L_{n-1}, G\right) \oplus \operatorname{Hom}\left(L_{n}, G\right)
$$

and with regards to the sequence $(S)$, we obtain a commutative diagram

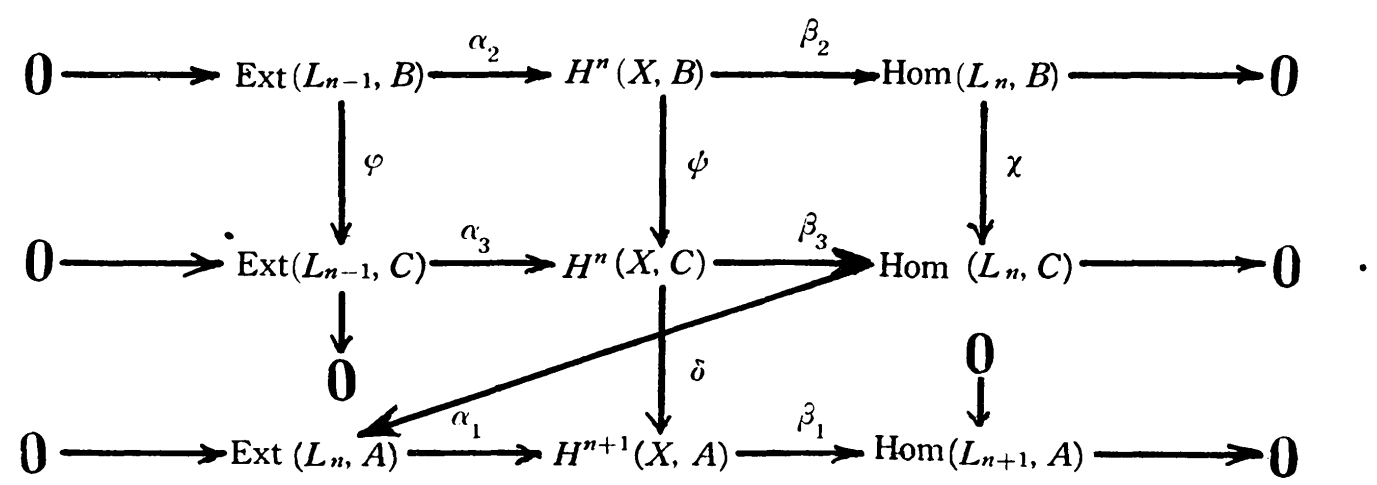

Diagram 2.

In this diagram (2), the $\delta$ kernel is the $\psi$ image by the exactness of the sequence

$$
H^{n}(X, B) \stackrel{\psi}{\longrightarrow} H^{n}(X, C) \stackrel{\delta}{\longrightarrow} H^{n+1}(X, A) \text {. }
$$

Since $\varphi$ is epimorphic by 1.5 , the $\varphi$ image of $H^{n}(X, B)$ contains the $\alpha_{3}$ image of $\operatorname{Ext}\left(L_{n}, C\right)$. Also, since $\beta_{2}$ is epimorphic, the $\beta_{3}$ image of $\psi H^{n}(X, B)$ coincides with the $\chi$ image of $\operatorname{Hom}\left(L_{n}, B\right)$. Noting that $\beta_{3}$ kernel is the $\alpha_{3}$ image of $\operatorname{Ext}\left(L_{n-1}, C\right)$, we obtain

$$
\left(H^{n}(X, C) \supset\right) \delta^{-1}(0) \approx \operatorname{Ext}\left(L_{n-1}, C\right) \oplus \chi \operatorname{Hom}\left(L_{n}, B\right) .
$$

Next, since the $\beta_{3}$ image of $\psi H^{n}(X, B)$ coincides with the $\chi \circ \beta_{2}$ image of $H^{n}(X, B)$ and is contained in the $\chi$ image of $\operatorname{Hom}\left(L_{n}, B\right), \beta_{3}$ induces the homomorphism

$$
\beta_{3}^{*}: H^{n}(X, C) / \psi H^{n}(X, B) \rightarrow \operatorname{Hom}\left(L_{n}, C\right) / \chi \operatorname{Hom}\left(L_{n}, B\right) .
$$

$\beta_{3}^{*}$ is epimorphic, for $\beta_{3}$ is epimorphic. We shall show that $\beta_{3}^{*}$ is a monomorphism. Let $b$ and $c$ be respectively elements of $\operatorname{Hom}\left(L_{n}, B\right)$ and $H^{n}(X, C)$ such that $\beta_{3}(c)=\chi(b)$. As $\beta_{2}$ is epimorphic, there exists 
an element $b^{\prime}$ of $H^{n}(X, B)$ with $\beta_{2}\left(b^{\prime}\right)=b$. So we have $\beta_{3} \circ \psi\left(b^{\prime}\right)=\chi \circ \beta_{2}\left(b^{\prime}\right)$ $=\beta_{3}(c)$. Therefore there is an element $c^{\prime} \in \operatorname{Ext}\left(L_{n-1}, C\right)$ with $\alpha_{3}\left(c^{\prime}\right)$ $=\psi\left(b^{\prime}\right)=c$. Furthermore $\varphi$ is epimorphic, and so we have an element $b^{\prime \prime}$ in $\operatorname{Ext}\left(L_{n-1}, B\right)$ such that $\varphi\left(b^{\prime \prime}\right)=c^{\prime}$. Then it follows from $\alpha_{3} \circ \varphi\left(b^{\prime \prime}\right)$ $=\varphi \circ \alpha_{2}\left(b^{\prime \prime}\right)=\varphi\left(b^{\prime}\right)-c$ that $c=\varphi\left(b^{\prime}-\alpha_{2}\left(b^{\prime \prime}\right)\right)$. This shows that $\beta_{3}^{*}$ is monomorphic. Hence we obtain:

THEOREM 2.1. Let .

$$
0 \longrightarrow A \longrightarrow B \stackrel{g}{\longrightarrow} C \longrightarrow 0
$$

be an exact sequence, and $\delta$ be the coboundary operator associated with this sequence. Then we have

$$
\begin{aligned}
& \left(H^{n}(X, C) \supset\right) \delta^{-1}(0) \approx \operatorname{Ext}\left(L_{n-1}, C\right) \oplus g \operatorname{Hom}\left(L_{n}, B\right) \\
& \delta H^{n}(X, C) \approx \operatorname{Hom}\left(L_{n}, C\right) / g \operatorname{Hom}\left(L_{n}, B\right), \quad\left(L_{i}=H_{i}(X, Z)\right) .
\end{aligned}
$$

REMARK 2.2. In the diagram (1) $\delta H^{n}(X, C)$ is the $\lambda$ image of $\operatorname{Hom}\left(L_{n}, C\right)$.

REMARK 2.3. As to the boundary operator $\delta_{*}$ in homology groups associated with the above sequence $(S)$, we have

$$
\begin{gathered}
\left(H_{n}(X, C) \supset\right) \delta_{*}^{-1}(0) \approx g\left(L_{n} \otimes B\right) \oplus L_{n-1} * C, \\
\delta_{*} H_{n}(X, C) \approx L_{n} \otimes C / g\left(L_{n} \otimes B\right) .
\end{gathered}
$$

ExAmPle 2.4. Let $(S)$ be the exact sequence

$$
0 \longrightarrow Z_{p} \stackrel{f_{h}^{\prime}}{\longrightarrow} Z_{p^{h+1}} \stackrel{g_{h}^{\prime}}{\longrightarrow} Z_{p^{h}} \longrightarrow 0 \text {. }
$$

Then we have

$$
\delta_{h}^{\prime} H^{n}\left(X, Z_{p^{h}}\right) \approx \operatorname{Hom}\left(L_{n}, Z_{p^{h}}\right) / g_{h}^{\prime} \operatorname{Hom}\left(L_{n}, Z_{p^{h+1}}\right) \text {. }
$$

Suppose that $H_{*}(X, Z)$ is of finite type in all degrees, then $L_{n}$ admits a direct sum decomposition into cyclic groups. If the number of summands in this decomposition whose orders are powers of $p$ with exponents $\leqq h$ is exactly $n^{\prime}$, then our $\delta_{h}^{\prime}$ image is a vector space with dimension $n^{\prime}$ over $Z_{l}$.

In the same way, we have

$$
\left(H_{n}\left(X, Z_{p^{h}} \supset\right) \delta_{h}^{\prime-1}(0) \approx \operatorname{Ext}\left(L_{n-1}, Z_{p}\right) \oplus g_{h}^{\prime} \operatorname{Hom}\left(L_{n}, Z_{p^{h+1}}\right) .\right.
$$

In particular $\delta_{1}^{\prime-1}(0)\left(\subset H^{n}\left(X, Z_{p}\right)\right)$ is a vector space with the same 
dimension as (Tor $\left.L_{n-1} \otimes Z_{p}\right) \oplus\left(\right.$ the free part of $\left.\left.L_{n}\right) \otimes Z_{p}\right) \oplus\left(\left(\sum\right.\right.$ the cyclic direct summands of order $p^{\nu}(\nu \geqq 2)$ of $\left.\left.L_{n}\right) \otimes Z_{p}\right)$ over $Z_{p^{*}}$ Namely $\delta_{1}^{\prime-1}$ (0) is the so-called $\left(\frac{1}{p} \delta \bmod p\right)$ kernel.

EXAMPLE 2.5. Let $(S)$ be the exact sequence

$$
0 \longrightarrow Z_{p} \stackrel{f_{h}}{\longrightarrow} Z_{p^{h+1}} \stackrel{g_{h}}{\longrightarrow} Z_{p} \longrightarrow 0 \text {. }
$$

Then we have

$$
\left(H^{n}\left(X, Z_{p}\right) \supset\right) \delta_{h}^{-1}(0) \approx \operatorname{Ext}\left(L_{n-1}, Z_{p}\right) \oplus g_{h} \operatorname{Hom}\left(L_{n}, Z_{p^{h+1}}\right) .
$$

Hence follows that $\delta_{h}^{-1}(0)$ has the same dimension as $\left(\sum\right.$ the $p$-primary direct summands of $\left.\left.L_{n-1}\right) \otimes Z_{p}\right) \oplus\left(\left(\right.\right.$ the free part of $\left.\left.L_{n}\right) \otimes Z_{p}\right) \oplus\left(\left(\sum\right.\right.$ the cyclic direct summands of order $\left.\left.p^{\nu}(\nu \geqq h+1)\right) \otimes Z_{p}\right)$.

Of course $\delta_{1}^{-1}(0)$ is equal to $\delta_{1}^{\prime-1}(0) . \quad \delta_{h} H^{n}\left(X, Z_{p}\right)$ is of dimension $n^{\prime}$ (see Example 2.4).

EXAMPLE 2.6. Consider the coboundary operator $\frac{1}{p} \delta$ associated with the exact sequence

$$
0 \longrightarrow Z \longrightarrow Z \longrightarrow Z_{p} \longrightarrow 0
$$

Then $\frac{1}{p} \delta$ kernel has the same dimension as $\left(\left(\sum\right.\right.$ the $p$-primary direct summands of $\left.\left.L_{n-1}\right) \otimes Z_{p}\right) \oplus\left(\right.$ the free part of $\left.\left.L_{n}\right) \otimes Z_{p}\right)$.

The $\frac{1}{p} \delta$ image has the same dimension as $\left(\sum\right.$ the $p$-primary direct summands of $\left.L_{n}\right) \otimes Z_{p}$.

PROPOSITION 2.7. The coboundary operator $\delta$ associated with the exact sequence

(S)

$$
0 \longrightarrow A \stackrel{f}{\longrightarrow} B \stackrel{g}{\longrightarrow} C \longrightarrow 0
$$

commutes with the coboundary homomorphism $\Delta$ of the cohomology sequence up to sign:

$$
\delta \Delta=-\Delta \delta .
$$

ProOF. For an exact sequence of chain complexes consisting of free groups, 


$$
0 \longleftarrow{ }^{\lambda} \stackrel{\lambda}{\longleftarrow} F \stackrel{\mu}{\longleftarrow} Q \longleftarrow 0,
$$

we have for any group $G$

$$
0 \longrightarrow C^{i}(R, G) \stackrel{\lambda^{*}}{\longrightarrow} C^{i}(F, G) \stackrel{\mu^{*}}{\longrightarrow} C^{i}(Q, G) \longrightarrow 0,
$$

where $C^{i}(R, G), C^{i}(F, G), C^{i}(Q, G)$ denote the $i$-th cochain groups with the coefficient $G$. Furthermore commutativity holds in the following diagram :

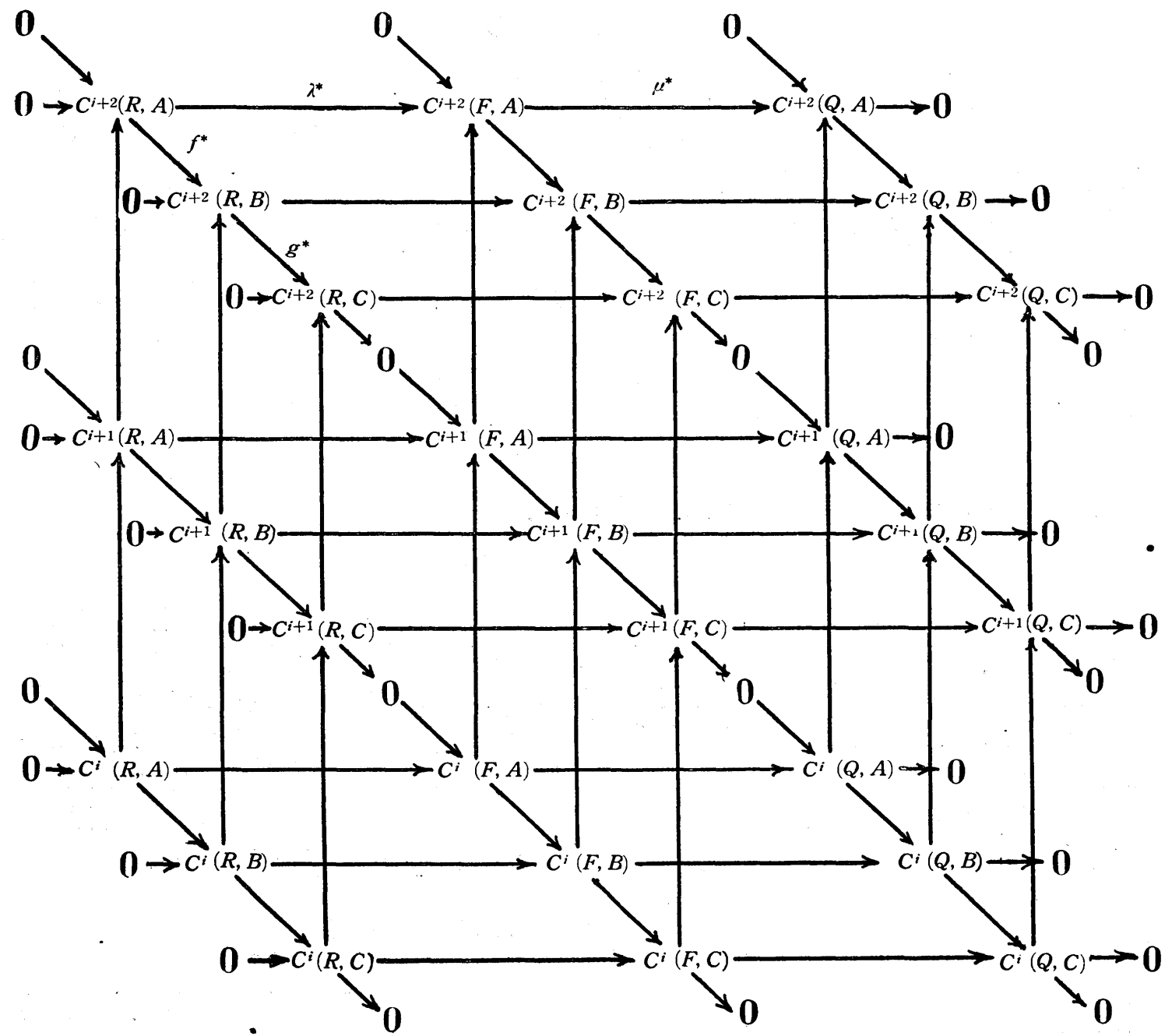

Diagram 3.

We shall denote by $d$ any of the coboundary operators in any of 
the above cochain groups unless no confusion is likely to occur. Take a cocycle $q_{c}^{i}$ of $C^{i}(Q, C)$. Then as $\mu^{*}$ is epimorphic, there exists an element $f_{c}^{i} \in C^{i}(F, C)$ such that the $\mu^{*}$ image of $f_{c}^{i}$ is $q_{c}^{i}$. Take $f_{c}^{i+1}=$ $d\left(f_{c}^{i}\right)$. This is annuled by $\mu^{*}$. Therefore there exists an element $r_{c}^{i+1}$ of $C^{i+1}(R, C)$ whose $\lambda^{*}$ image is $f_{c}^{i+1}$. Further $g$ is epimorphic, and so we have $g\left(r_{b}^{i+1}\right)=r_{c}^{i+1}$ with some $r_{b}^{i+1} \in C^{i+1}(R, B)$. On the other hand, we have

$$
g \circ d\left(r_{b}^{i+1}\right)=d \circ g\left(r_{b}^{i+1}\right)=0 \text {. }
$$

This implies the existence of $r_{a}^{i+2} \in C^{t+2}(R, A)$ such that

$$
f^{*}\left(r_{a}^{i+2}\right)=d\left(r_{b}^{i+1}\right) \text {. }
$$

Now $g$ and $\mu^{*}$ being epimorphic, there exists an element $f_{b}^{i} \in$ $C^{i}(F, B)$ whose $g$ image is $f_{c^{*}}^{i}$ Since

$$
g\left(d f_{b}^{i}-\lambda^{*} r_{b}^{i+1}\right)=d \circ g\left(f_{b}^{i}\right)-\lambda^{*} \circ g\left(r_{b}^{i+1}\right)=f_{c}^{i+1}-f_{c}^{i+1}=0,
$$

there is an element $f_{a}^{i+1}$ of $C^{i+1}(F, A)$ whose $f^{*}$ image is $d\left(f_{b}^{i}\right)-\lambda^{*}\left(r_{b}^{i+1}\right)$. Then we have

$$
f_{\circ} \mu^{*}\left(f_{a}^{i+1}\right)=\mu^{*} \circ\left(d\left(f_{b}^{i}\right)-\lambda^{*}\left(r_{b}^{i+1}\right)\right)=\mu^{*} \circ d\left(f_{b}^{i}\right)=d \circ \mu^{*}\left(f_{b}^{i}\right) .
$$

Moreover we have

$$
\begin{aligned}
f\left(d\left(f_{a}^{i+1}\right)+\lambda^{*}\left(r_{a}^{i+2}\right)\right)=d \circ f\left(f_{a}^{i+1}\right)+\lambda^{*} \circ f\left(r_{a}^{i+2}\right) \\
=-d \circ \lambda^{*}\left(r_{b}^{i+1}\right)+\lambda^{*} \circ d\left(r_{b}^{i+1}\right)=0
\end{aligned}
$$

and $f^{*}$ is monomorphic. Therefore we have $d\left(f_{a}^{i+1}\right)=-\lambda^{*}\left(r_{a}^{i+1}\right)$, also we have $\mu^{*} \circ d\left(f_{a}^{i+1}\right)=0$. Therefore there exists $r_{a}^{i+2}=C^{t+2}(R, A)$ with the property $\lambda^{*}\left(r_{a}^{i+2}\right)=d\left(f_{a}^{i+1}\right)\left(=\lambda^{*}\left(-r_{a}^{i+2}\right)\right)$. That is $r_{a}^{i+2}=-r_{a}^{i+2}$ as $\lambda^{*}$ is epimorphic. This implies that the cohomology class $\left\{\boldsymbol{r}_{a}^{i+2}\right\}$ of $\boldsymbol{r}_{a}^{i+2}$ representing $\Delta \circ \delta\left\{\boldsymbol{q}_{c}^{i}\right\}$ is the same as the cohomology class $-\left\{\boldsymbol{r}_{a}^{i+2}\right\}$ of $\boldsymbol{r}_{a}^{i+2}$ representing $-\delta \circ \Delta\left\{\boldsymbol{q}_{c}^{i}\right\}$.

Q. E. D.

PROPOSITION 2.9. In the exact sequence of groups

$$
0 \longrightarrow \stackrel{i}{\longrightarrow} B \stackrel{k}{\longrightarrow} B / i A \longrightarrow 0 \text {, }
$$

we assume that a commutative ring structure is given in each of $A, B$, $B / i A$ in such a way that

(i) $k$ is a ring homomorphism and

(ii) for the ideal iA in $B$, we have $(i A)^{2}=0$. 
Then we can define a bilinear multiplication of $A$ and $B / i A$ into $A$ induced by the natural multiplication of $i A$ and $B$, whence a bilinear pairing of $H^{*}(X, A)$ and $H^{*}(X, B / i A)$ can be defined in an obvious way.

Let $\delta$ be the coboundary operator associated with the above exact sequence. For $\alpha \in H^{\prime}(X, B / i A), \beta \in H^{j^{\prime}}(X, B / i A)$, we have then the following equality

$$
\delta(\alpha \beta)=\delta(\alpha) \beta+(-\mathbf{1})^{j} \alpha \delta(\beta) .
$$

Proof is left to the reader.

PROPOSITION 2.10. The coboundary operator $\delta_{h}^{\prime}$ is obtained in composing the coboundary operator associated with the exact sequence

$$
0 \longrightarrow Z \stackrel{F_{h}}{\longrightarrow} Z \stackrel{G_{h}}{\longrightarrow} Z_{p} \longrightarrow 0
$$

with the homomorphism $H^{*}(X, Z) \rightarrow H^{*}\left(X, Z_{p}\right)$ induced by the natural homomorphism $Z \rightarrow Z_{p}(\rightarrow 0)$, and the coboundary operator $\delta_{h}$ is obtained in composing the coboundary operator associated with the exact sequence

$$
0 \longrightarrow Z \stackrel{F_{1}}{\longrightarrow} Z \stackrel{G_{1}}{\longrightarrow} Z \longrightarrow 0
$$

with the homomorphism $H^{*}(X, Z) \rightarrow H^{*}\left(X, Z_{p^{h}}\right)$ induced by the natural homomorphism $Z \rightarrow Z_{p} h(\rightarrow 0)$.

PROOF. It follows from the following commutative diagram:

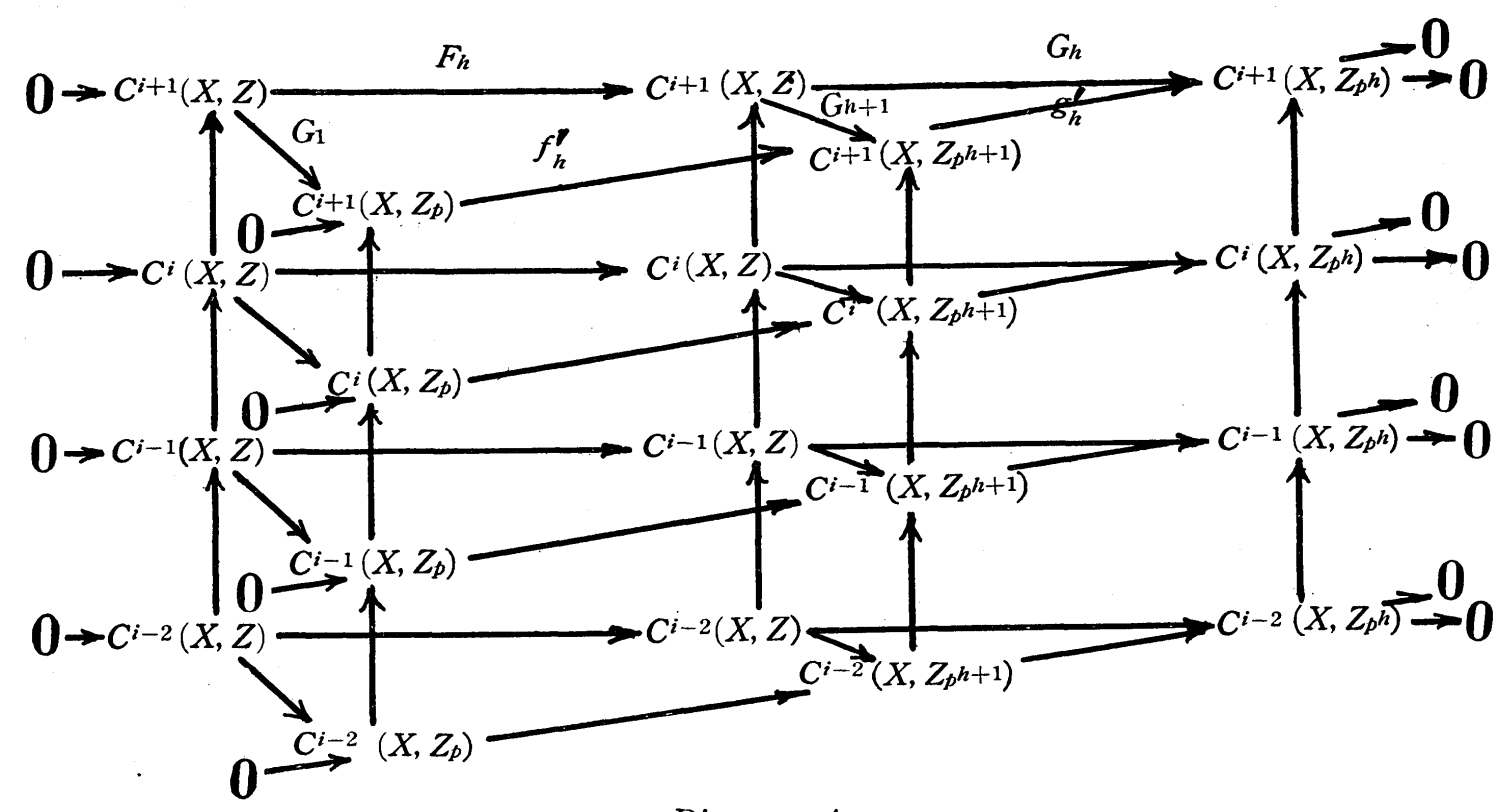

Diagram 4. 


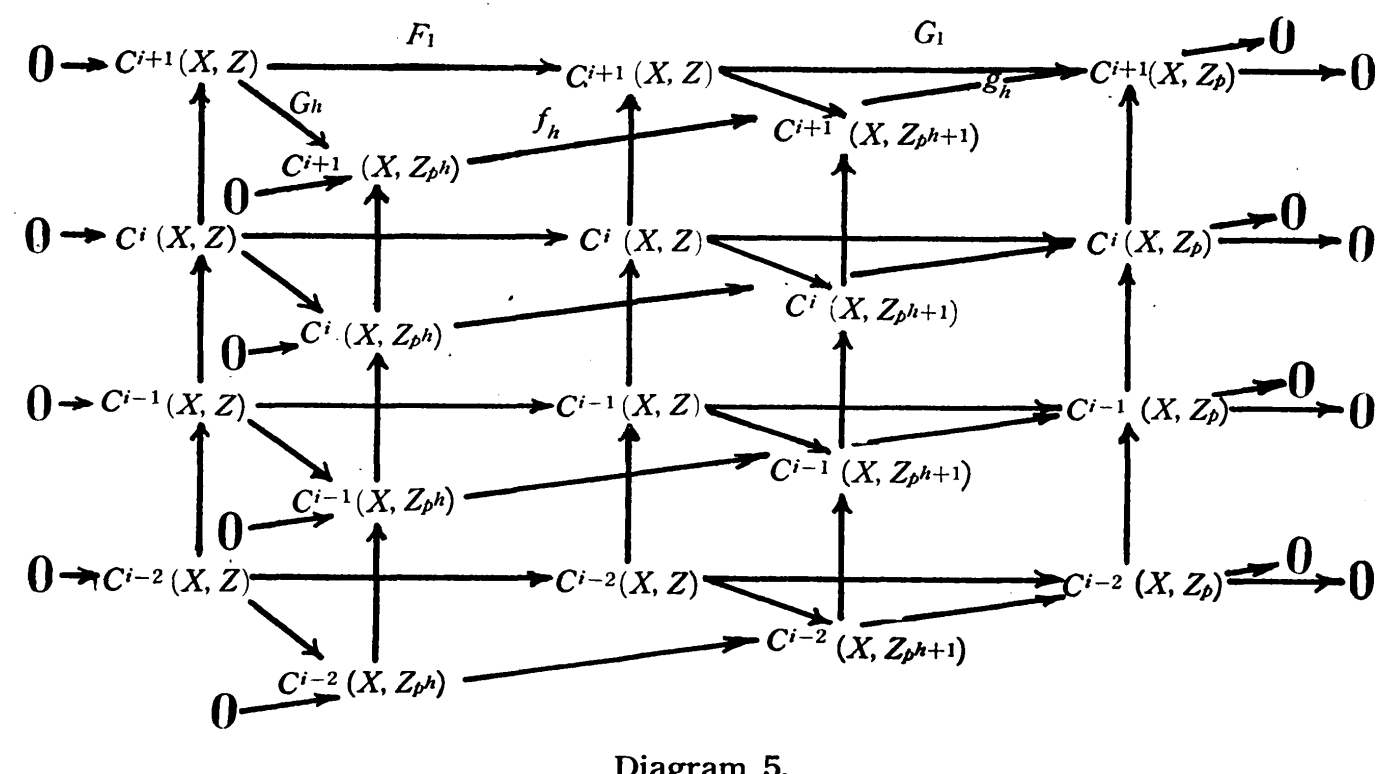

Diagram 5.

\section{$\S 2$. Definition of $\Delta_{p}^{i}$ and its properties}

1. Definition of $\Delta_{p}^{i}$. The cohomological operations $\Delta_{p}^{i}(i=1,2, \cdots)$ is inductively defined as follows.

Operation $\Delta_{p}^{1}$ is defined as the coboundary operator associated with the exact sequence

$$
0 \longrightarrow Z_{p} \longrightarrow Z_{p^{2}} \longrightarrow Z_{p} \longrightarrow 0 \text {. }
$$

Thus $\Delta_{p}^{1}$ is nothing else than $\delta_{1}$ of Example $\S 1,2.5$.

Assume the operation $\Delta_{p}^{i}$ are defined $i=1, \cdots, h-1,(h>1)$ and the $\Delta_{p}^{h-1}$ kernel coincides with the $\delta_{h-1}$ kernel. Then we shall define as follows

$$
\Delta_{p}^{h}: \Delta_{p}^{h-1} \text { kernel }\left(\subset H^{n-1}\left(X, Z_{p}\right)\right) \rightarrow H^{n}\left(X, Z_{p}\right) \text { mod } \delta_{h-1}^{\prime} \text { image. }
$$

Obviously we have the following commutative diagram: 


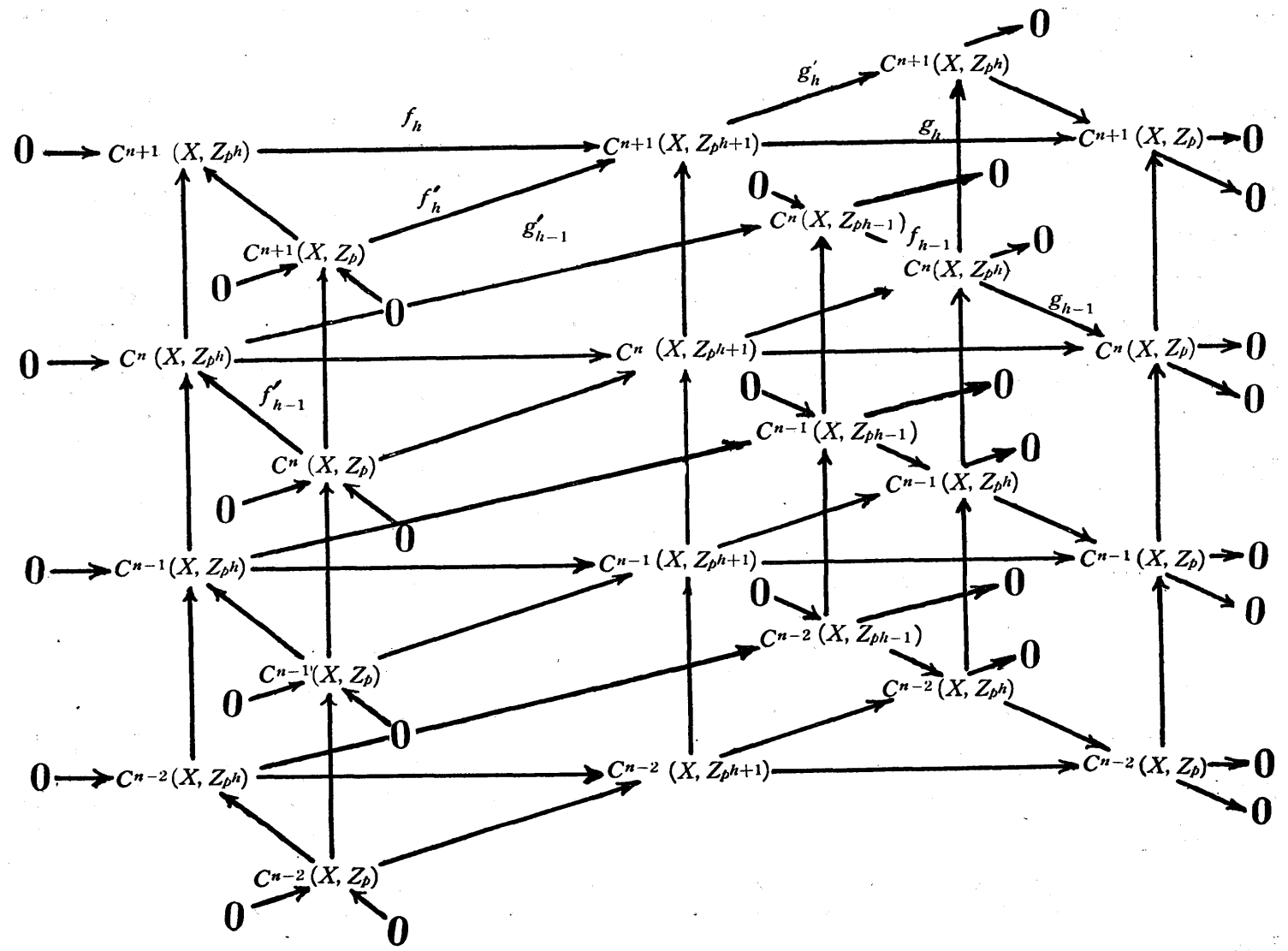

Diagram 6.

Let $c$ be an element of $H^{n-1}\left(X, Z_{p}\right)$ whose $\Delta_{p}^{h-1}$ image is zero. Then by the exactness of the following sequence

$$
\begin{aligned}
\cdots \longrightarrow H^{n-1}\left(X, Z_{p^{h-1}}\right) \stackrel{f_{h-1}}{\longrightarrow} & H^{n-1}\left(X, Z_{\left.p^{h}\right) \stackrel{g_{h-1}}{\longrightarrow}}^{\longrightarrow} H^{n-1}\left(X, Z_{p}\right) \stackrel{\delta_{h-1}}{\longrightarrow}\right. \\
& H^{n}\left(X, Z_{p^{h-1}} \longrightarrow \cdots,\right.
\end{aligned}
$$

c is contained in the $g_{n-1}$ image of $H^{n-1}\left(X, Z_{p} h\right)$. That is, take a representative cocycle $c$ of class $c$, then there exists a cocycle $c_{1} \in$ $C^{n-1}\left(X, Z_{p^{h}}\right)$ such that $g_{h-1}\left(c_{1}\right)=c . \quad g_{h}^{\prime}$ is epimorphic, and so we have $\boldsymbol{g}_{h}^{\prime}\left(c_{2}\right)=c_{1}$ for some cochain $c_{2}$ of $C^{n-1}\left(X, Z_{p^{h+1}}\right)$. Then $g_{h}^{\prime} \circ \delta\left(c_{2}\right)=\delta \circ g_{h}^{\prime}\left(c_{2}\right)$ $=\delta\left(c_{1}\right)=0$. Since $f_{h}^{\prime}$ is monomorphic, there exists a unique element $c_{3}$ of $C^{n}\left(X, Z_{p}\right)$ such that $f_{h}^{\prime}\left(c_{3}\right)=\delta\left(c_{2}\right)$. Clearly $c_{3}$ is a cocycle. Then $\Delta_{p}^{h}(\mathfrak{c})$ is defined as the cohomology class of mod. $\delta_{h-1}^{\prime}$ image. The class $\left\{c_{3}\right\}$ does not depend on the choice of $c_{2}$ out of $C^{n-1}\left(X, Z_{p^{h+1}}\right)$, and, of course on the choice of $c$, but depends on the class $c_{1}$. $c$ beeing given, 
we can replace $\left\{c_{1}\right\}$ by $\left\{c_{1}+c_{1}^{\prime}\right\}$, where $c_{1}^{\prime}=f_{h-1}\left(c_{1}^{\prime \prime}\right), c_{1}^{\prime \prime}$ being a cocycle in $C^{n-1}\left(X, Z_{p^{h-1}}\right)$. Since $g_{h-1}^{\prime}$ is epimorphic, there exists a cochain $c_{2}^{\prime \prime} \in C^{n-1}\left(X, Z_{p^{h}}\right)$ whose $g_{h-1}^{\prime}$ image coincides with $\boldsymbol{c}_{1}^{\prime \prime}$. Since $\boldsymbol{g}_{n-1}^{\prime} \circ \delta\left(\boldsymbol{c}_{2}^{\prime \prime}\right)$ $=0$, there is an element $c_{3}^{\prime \prime} \in C^{n}\left(X, Z_{p}\right)$ whose $f_{h-1}^{\prime}$ image is $\delta\left(c_{2}^{\prime \prime}\right)$. Thus the $\delta_{h-1}^{\prime}$ image of the class $\left\{c_{1}^{\prime}\right\}$ is the class $\left\{c_{3}^{\prime \prime}\right\}$. This completes the definition of the operation $\Delta_{i}^{h}$.

Furthermore we prove now that the $\Delta_{p}^{h}$ kernel is the $\delta_{h}$ kernel. First, let us show the $\delta_{h}$ kernel is contained in the $\Delta_{p}^{h}$ kernel. Let $c$ be a cocycle of $C^{n-1}\left(X, Z_{p}\right)$ such that $\delta_{h}(\{c\})=0$, then there exists a cocycle $c^{\prime} \in C^{n-1}\left(X, Z_{p^{h-1}}\right)$ with $g_{h}\left(c^{\prime}\right)=c$ by the exactness of the following sequence

$$
\begin{gathered}
\cdots \longrightarrow H^{n-1}\left(X, Z_{p^{h}}\right) \stackrel{f_{h}}{\longrightarrow} H^{n-1}\left(X, Z_{\left.p^{h+1}\right)} \stackrel{g_{h}}{\longrightarrow} H^{n-1}\left(X, Z_{p}\right) \longrightarrow\right. \\
H^{n}\left(X, Z_{p^{h}}\right) \longrightarrow \cdots .
\end{gathered}
$$

As $\delta\left(c^{\prime}\right)=0$, we have $\Delta_{p}^{h}(\{c\})=0$.

Second, we prove that the $\Delta_{p}^{h}$ kernel is contained in the $\delta_{h}$ kernel. In using the above notations, we may write $c_{3}=c_{3}^{\prime \prime}+\delta(b), b \in c^{n-1}\left(X, Z_{p}\right)$.

Then we have

$$
f_{h}^{\prime}\left(c_{3}\right)=\delta\left(c_{2}\right)=f_{h}^{\prime}\left(c_{3}^{\prime \prime}\right)+f_{h}^{\prime} \circ \delta(b)=\delta \circ f_{h}\left(c_{2}^{\prime \prime}\right)+\delta \circ f_{h}^{\prime}(b),
$$

hence

$$
\delta\left(c_{2}-f_{h}\left(c_{2}^{\prime \prime}\right)-f_{h}^{\prime}(b)\right)=0
$$

Clearly

$$
\begin{aligned}
& g_{h}\left(c_{2}-f_{h}\left(c_{2}^{\prime \prime}\right)-f_{h}^{\prime}(b)\right) \\
= & g_{h-1} \circ g_{h}^{\prime}\left(c_{2}\right)-g_{h} \circ f_{h}\left(c_{2}^{\prime \prime}\right)-g_{h} \circ f_{h} \circ f_{h-1}^{\prime}(b)=c .
\end{aligned}
$$

This implies $\delta_{h}\{c\}=0$.

Thus the cohomological operations $\Delta_{p}^{i}$ are now defined for all $i=$ $1,2,3, \cdots$.

From the definition of $\Delta_{p}^{h}$ and $\S 1$ Example 2.4, we have

THEOREM 1.1. Let $H_{*}(X, Z)$ be of finite type in all degrees, and $n^{\prime}$ be the number of cyclic direct summands of $H_{n-1}(X, Z)$ with order $p^{i}$. Then the $\Delta_{p}^{i}$ image in $H^{n}\left(X, Z_{p}\right) \bmod \delta_{i-1}^{\prime}$ image has the dimension $n^{\prime}$ over $Z_{p}$. The $\Delta_{p}^{i}$ kernel is equal to the $\delta_{i}$ kernel.

REMARK 1.2. The operations $\Delta_{p}^{i}$ can be defined in relative cohomology groups in the same manner. 
REMARK 1.3. Similarly we can define corresponding operations in homology groups.

REMARK 1.4. We can define $\Delta_{p^{\lambda}}^{1}$ as the coboundary operator associated with the exact sequence

$$
0 \longrightarrow Z_{p^{\lambda}} \longrightarrow Z_{p^{2 \lambda}} \longrightarrow Z_{p^{\lambda}} \longrightarrow 0,
$$

and define inductively $\Delta_{p^{\lambda}}^{i}(i=2,3, \cdots)$ similarly as above.

2. The fundamental properties of the operations $\Delta_{p}^{i}$

From the definition of $\Delta_{p}^{i}$ follows immediately

2.1. The operations $\Delta_{p}^{i}$ commute with the homomorphism of cohomology groups induced by mapping of spaces:

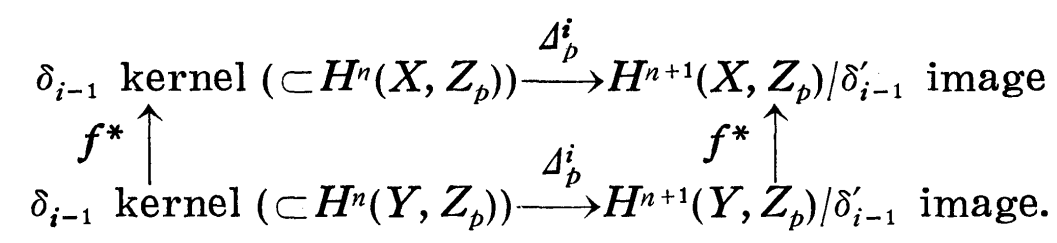

From Proposition 2.7 in $\S 1$ follows further

2.2. The operations $\Delta_{p}^{i}$ commutes with the coboundary homomorphism $\Delta$ of cohomology sequence up to sign:

2.1 and 2.2 imply

$$
\Delta_{p}^{i} \circ \Delta=-\Delta \circ \Delta_{p}^{i} \text {. }
$$

2.3. The operations $\Delta_{p}^{i}$ commute with the transgression $\tau$ up to sign :

$$
\Delta_{p}^{i} \circ \tau=-\tau \circ \Delta_{p}^{i}
$$

Now we have

2.4. $\Delta_{p}^{j} \circ \Delta_{p}^{i}$ has the natural meaning and is

$$
\Delta_{p}^{j} \circ \Delta_{p}^{i}=0 \text {. }
$$

Especially

$$
\Delta_{p}^{i} \circ \Delta_{p}^{i}=0 \text {. }
$$

Since $\Delta_{p}^{i}$ image $=\delta_{i}^{\prime}$ image $/ \delta_{i-1}^{\prime}$ image, this follows from the following Lemma.

LEMMA 2.5. $\quad \delta_{j} \circ \delta_{i}^{\prime}=0$.

ProOF. The $\delta_{i}^{\prime}$ image of $H^{n-1}\left(X, Z_{p^{i}}\right)$ is contained in the $G_{1}$ image of $H^{n}(X, Z)$ by $\S 1$, Prop. 2.10 and the $G_{1}$ image of $H^{n}(X, Z)$ is contained in the $g_{j}$ image of $H^{n}\left(X, Z_{p}{ }^{j+1}\right)$ by $G_{1}=g_{j} \circ G_{j+1}$. Then $\delta_{j} \circ \delta_{i}^{\prime}=0$ follows immediately from the exactness of the following sequence. 


$$
H^{n}\left(X, Z_{p^{j-1}}\right) \stackrel{g_{j}}{\longrightarrow} H^{n}\left(X, Z_{p}\right) \stackrel{\delta_{j}}{\longrightarrow} H^{n+1}\left(X, Z_{p^{j}}\right) .
$$

We have furthermore

2.6. Let $\alpha$ and $\beta$ be respectively elements of $H^{m}\left(X, Z_{p}\right)$ and $H^{n}\left(X, Z_{p}\right)$ satisfying $\delta_{i-1}(\alpha)=\delta_{i-1}(\beta)=0$, then $\Delta_{p}^{i}(\alpha \beta)$ is definable, as $\delta_{i-1}(\alpha \beta)=0$ follows from the assumption $\delta_{i-1}(\alpha)=\delta_{i-1}(\beta)=0$. That $\Delta_{p}^{i}(\alpha) \beta$ and $\alpha \Delta_{p}^{i}(\beta)$ have the natural meanings follows from

$$
\left(\delta_{i-1} \text { kernel) }\left(\delta_{i-1}^{\prime} \text { image }\right) \subset \delta_{i-1}^{\prime}\right. \text { image. }
$$

Finally the equality

$$
\Delta_{p}^{i}(\alpha \beta)=\Delta_{p}^{i}(\alpha) \beta+(-1)^{m} \alpha \Delta_{p}^{i}(\beta),
$$

follows from $\S 1$ Proposition 2.9.

Let us put $\delta_{h}$ kernel $=J_{h}, \delta_{h}^{\prime}$ image $=J_{h}^{\prime}(h=1,2, \cdots) \underset{h}{\cup} J_{h}^{\prime}=J_{\infty}^{\prime}, \bigcap_{h} J_{h}=J_{\infty}$. Then we have

Proposition 2.7.

$$
J_{1}^{\prime} \subset J_{2}^{\prime} \subset \cdots \subset J_{h}^{\prime} \subset \cdots \subset J_{\infty}^{\prime} \subset J_{\infty} \subset \cdots \subset J_{h} \subset \cdots J_{2} \subset J_{1} .
$$

PROOF. $J_{h}=\delta_{h}$ kernel $\supset \delta_{h+1}$ kernel $=J_{h+1}$ is clear from the definition of the operations $\Delta_{p}^{i}$.

Now let $u$ be a cocycle of $C^{n-1}\left(X, Z_{p^{h}}\right)$, then the $\delta_{h}^{\prime}$ image of the class $\{\boldsymbol{u}\}$ is equal to the $\delta_{h+1}^{\prime}$ image of the class $\left\{f_{h}(u)\right\}$. (See diagram (5)). Hence follows $J_{h+1}^{\prime}=\delta_{h+1}^{\prime}$ image $\supset \delta_{h}^{\prime}$ image $=J_{h}^{\prime}$.

PROPOSITION 2.8. We have
(1) if $h \leqq k$
$J_{h} \cdot J_{k} \subset J_{h}$
(3) if $h \leqq k$ $J_{h}^{\prime} \cdot J_{k} \subset J_{k} \quad$ for arbitrary $h, k$,
(4) if $h \leqq k$ $J_{h}^{\prime} \cdot J_{k} \subset J_{h}^{\prime}$,

Proof. (1) follows from 2.6 and Theorem 1.1, and (2) from (1) and Proposition 2.7.

As for (3), let $\alpha^{\prime \prime}, \alpha^{\prime}, \alpha$ and $\beta$ be respectively a cocycle of $C^{r}\left(X, Z_{p} h\right)$, a cochain of $C^{r}\left(X, Z_{p^{h+1}}\right)$, a cochain of $C^{r+1}\left(X, Z_{p}\right)$ and a cocycle of $C^{s}\left(X, Z_{p^{k}}\right)$ such that $g_{h}^{\prime}\left(\alpha^{\prime}\right)=\alpha^{\prime \prime}, f_{h}^{\prime}(\alpha)=\delta\left(\alpha^{\prime}\right)$ and $\delta_{k}(\{\beta\})=0$. Then $\beta$ is the $g_{k}$ image of $\lambda$ which is a cocycle, by the exactness of the sequence

$$
\cdots \longrightarrow H\left(X, Z_{p^{k}}\right) \stackrel{f_{k}}{\longrightarrow} H^{s}\left(X, Z_{p^{k+1}}\right) \stackrel{g_{k}}{\longrightarrow} H^{s}\left(X, Z_{p}\right) \stackrel{\delta_{k}}{\longrightarrow} H^{s+1}\left(X, Z_{p^{k}}\right) \longrightarrow \cdots
$$


and by the commutativity of the following diagram

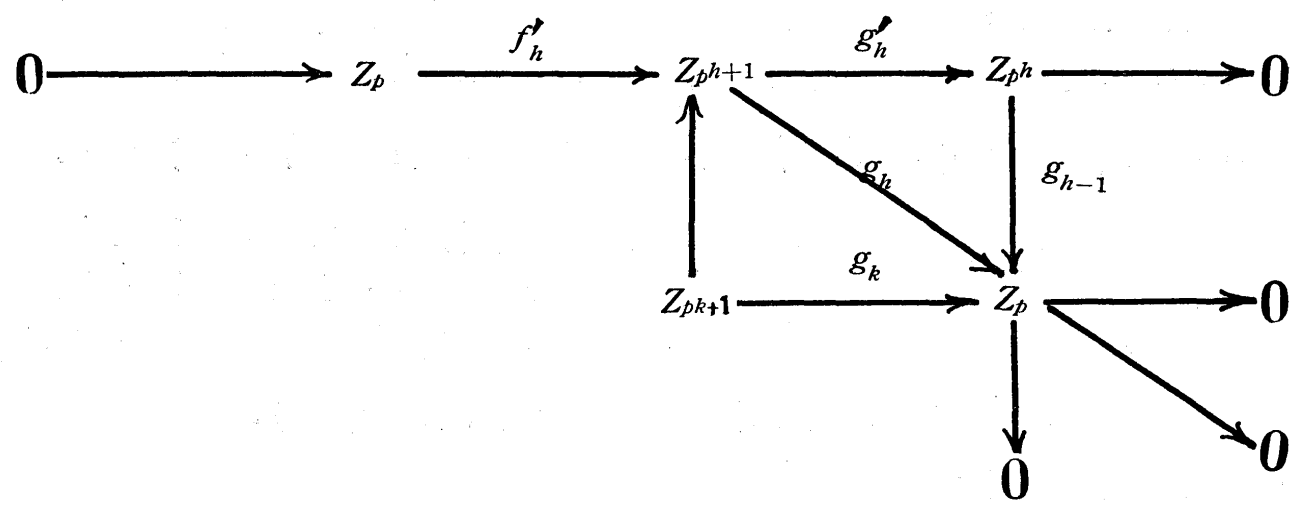

Diagram 7.

Take a cocycle $\alpha^{\prime \prime} g_{h}^{\prime}(\lambda)$ of $C^{r+s}\left(X, Z_{p^{h}}\right)$, then we have

$$
\alpha^{\prime \prime} g_{h}^{\prime}(\lambda)=g_{h}^{\prime}\left(\alpha^{\prime}\right) g_{h}^{\prime}(\lambda)=g_{h}^{\prime}\left(\alpha^{\prime} \lambda\right),
$$

and

$$
\begin{aligned}
\delta\left(\alpha^{\prime} \lambda\right) & =\delta\left(\alpha^{\prime}\right) \lambda+(-1)^{r} \alpha^{\prime} \delta(\lambda)=f_{h}^{\prime}(\alpha) \lambda \\
& =f_{h}^{\prime}\left(\alpha g_{h}(\lambda)\right)=f_{h}^{\prime}(\alpha \beta) .
\end{aligned}
$$

This completes the proof of (3), (4) follows immediately from (3), and Prop. 2.7

Q. E. D.

REMARK 2.9. The relations (1), (2), (3), (4) are also induced by a certain filtration of $C(X, Z)$.

From Proposition 2.8 follows

PROPOSITION 2.10. $J_{h} / J_{h}^{\prime}$ is an algebra over $Z_{p}$ with a differential operator $\bar{\Delta}_{p}^{h+1}$ for each $h$. Here $\bar{\Delta}_{p}^{h+1}$ is the operator induced by $\Delta_{p}^{h+1}$ and has a property $\bar{\Delta}_{p}^{h+1} \circ \bar{\Delta}_{p}^{h+1}=0$.

The structure of these algebras for Eilenberg-MacLane complex $K(\Pi, n)$ has been determined by T. Nakamura.

PROPOSITION 2.11. Let $v$ be an element of the $H^{r}\left(X, Z_{2}\right)$ with $\delta_{h-1}(v)=0 \quad\left(\delta_{0}\right.$ may be defined as the zero operator $)$, then we have

$$
\Delta_{2}^{h+1}\left(v^{2}\right)= \begin{cases}S q^{r} \circ \Delta_{2}^{1}(v)+v \Delta_{2}^{1}(v) & \text { if } r \text { is even and } h=1 \\ v \Delta_{2}^{h}(v) & \text { if } r \text { is even and } h>1 . \\ 0 & \text { if } r \text { is odd. }\end{cases}
$$

PROOF. If $r$ is odd, then $v^{2}=S q^{r} v=S q^{1} S q^{r-1} v=\Delta_{2}^{1}\left(S q^{r-1} v\right)$, and so the above result is clear by 2.4 . 
If $r$ is even, we have $\Delta_{2}^{h}\left(v^{2}\right)=0$ by 2.6. So we can compute $\Delta_{2}^{h+1}\left(v^{2}\right)$ as follows. Take a representative cocycle $v^{\prime}$ of $v$, then $v^{\prime}$ is the $g_{h-1}$ image of a cocycle $v^{\prime \prime} \in C^{r}\left(X, Z_{2} h\right)$ by the exactness of the sequence

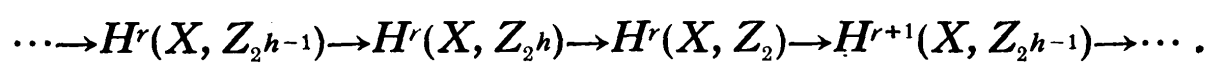

We consider a cochain $u \smile_{0} u+u \smile_{1} \delta(u)$, where $\smile_{i}$ is Steenrod's $i$-product, and $u$ is an integral cochain whose $G_{h}$ image is $v^{\prime \prime}$ (see $\S 1$ Proposition 2.10). Then Steenrod's coboundary formula shows that

$$
\delta\left(u \smile_{0} u+u \smile_{1} \delta(u)\right)=2 u \smile_{0} \delta(u)+\delta u \smile_{1} \delta(u) .
$$

Therefore we have

$$
\begin{aligned}
& F_{h+1}\left(u \smile_{0} F_{h}^{-1} \circ \delta(u)+2^{h-1} F_{h}^{-1} \circ \delta(u) \smile_{1} F_{h}^{-1} \circ \delta(u)\right) \\
= & 2 u \smile_{0} \delta(u)+\delta(u) \smile_{1} \delta(u),
\end{aligned}
$$

and

$$
\begin{aligned}
& G_{1}\left(u \smile_{0} F_{h}^{-1} \circ \delta(u)+2^{h-1} F_{h}^{-1} \circ \delta(u) \smile_{1} F_{h}^{-1} \circ \delta(u)\right) \\
= & \begin{cases}v^{\prime} \smile_{0} G_{1} \circ F_{h}^{-1} \circ \delta(u)+G_{1} \circ F_{h}^{-1} \circ \delta(u) \smile{ }_{1} G_{1} \circ F_{h}^{-1} \circ \delta(u) & \text { for } h=1 \\
v^{\prime} \smile_{0} G_{1} \circ F_{h}^{-1} \circ \delta(u) & \text { for } h>1 .\end{cases}
\end{aligned}
$$

This proves our Proposition.

REMARK 2.12. The above proof shows that $\delta_{2}^{\prime} \circ P_{2}^{1}(v) \bmod \delta_{1}^{\prime}$ image coincides with $\Delta_{2}^{2}\left(v^{2}\right)$, where $P_{2}^{1}(v)$ means the Pontryagin square of $v$. (See [11]).

PROPOSITION 2.13. Let $v$ be an element of $H^{r}\left(X, Z_{p}\right)$ with $\delta_{h-1}(v)=0$, and $p$ be an odd prime, then we have

$$
\Delta_{p}^{h+1}\left(v^{p}\right)=v^{p-1} \Delta_{p}^{h}(v) .
$$

Proof. Let $D_{i}$ be the Steenrod's $D_{i}$ operator. Then we have

$$
\begin{aligned}
& D_{i} \circ \delta(u)+(-1)^{i+1} \delta \circ D_{i}(u)=-D_{i-1}(u)+D_{i+1} \circ T(u) \text { if } i \text { is odd, } \\
& D_{i} \circ \delta(u)+(-1)^{i+1} \delta \circ D_{i}(u)=D_{i-1} \circ\left(1+T+\cdots+T^{p-1}\right)(u) \text { if } i \text { is even, }
\end{aligned}
$$

where $T(u)\left(\sigma_{1} \times \cdots \times \sigma_{p-1} \times \sigma_{p}\right)=(-1)^{D\left(\sigma_{p}\right)_{i=1}^{p-1} D\left(\sigma_{i}\right)} u\left(\sigma_{p} \times \sigma_{1} \times \cdots \times \sigma_{p-1}\right)$, and $D\left(\sigma_{j}\right)$ denotes the degree of $\sigma_{j}$.

We have only to consider the case, where $r$ is even. Then we have $\Delta_{p}^{h}\left(v^{p}\right)=0$ by 2.6. Let $v^{\prime}$ be a cochain such that $\left\{G_{1}\left(v^{\prime}\right)\right\}=v$ and $\delta\left(v^{\prime}\right)=\boldsymbol{p}^{h} \boldsymbol{v}^{\prime \prime}$. Then by (2) we have 


$$
\begin{aligned}
\delta \circ D_{0}\left(v^{\prime} \otimes \cdots \otimes v^{\prime}\right) & =D_{0} \circ \delta\left(v^{\prime} \otimes \cdots \otimes v^{\prime}\right) \\
& =\sum_{j_{1}=1}^{p} D_{0}\left(v^{\prime} \otimes \cdots \otimes \delta\left(v^{\prime}\right) \otimes \cdots \otimes v^{\prime}\right),
\end{aligned}
$$

where $\left(j_{1}\right)$ on $\delta\left(v^{\prime}\right)$ indicates that $\delta\left(v^{\prime}\right)$ is at the $j_{1}$-th place in the product $v^{\prime} \otimes \cdots \otimes \delta\left(v^{\prime}\right) \otimes \cdots \otimes v^{\prime}$. The same notation will be used in the following.

Also by (1) we have

$$
\begin{gathered}
D_{1} \circ \delta\left(v^{\prime} \otimes \cdots \otimes \delta\left(v^{\prime}\right) \otimes \cdots \otimes v^{\prime}\right)+\delta \circ D_{1}\left(v^{\prime} \otimes \cdots \otimes \delta\left(v^{\prime}\right) \otimes \cdots \otimes v^{\prime}\right) \\
\left\{\begin{array}{cc}
\left(j_{1}\right) \\
=-D_{0}\left(v^{\prime} \otimes \cdots \otimes \delta\left(v^{\prime}\right) \otimes \cdots \otimes v^{\prime}\right)+D_{0}\left(v^{\prime} \otimes \cdots \otimes \delta\left(v^{\prime}\right) \otimes \cdots \otimes v^{\prime}\right) \\
=-D_{0}\left(v^{\prime} \otimes \cdots \otimes \delta\left(v^{\prime}\right)\right)+D_{0}\left(\delta\left(v^{\prime}\right) \otimes v^{\prime} \cdots \otimes v^{\prime}\right) & \text { for } j_{1} \leqq p-1 \\
\text { for } j_{1}=p .
\end{array}\right.
\end{gathered}
$$

Furthermore the following equality holds

$$
\begin{aligned}
& D_{1} \circ \delta\left(v^{\prime} \otimes \cdots \otimes \delta^{\left(j_{1}\right)}\left(v^{\prime}\right) \otimes \cdots \otimes v^{\prime}\right)+\delta \circ D_{1}\left(v^{\prime} \otimes \cdots \otimes \delta\left({ }^{\left(j_{1}\right)}\right) \otimes \cdots \otimes v^{\prime}\right) \\
& =D_{1}\left(\sum_{1 \leqq j_{2}<j_{1}} v^{\prime} \otimes \cdots \otimes \delta \delta^{\left(j_{2}\right)}\right) \otimes \cdots \otimes \delta\left(v^{\prime}\right) \otimes \cdots\left(v^{\prime}\right) \otimes \cdots \otimes v^{\prime} \\
& \left.\left.-\sum_{j_{1}<j_{2} \leqq p} v^{\prime} \otimes \cdots \otimes \delta^{\left(j_{1}\right)}\left(v^{\prime}\right) \otimes \cdots \otimes\left({ }^{\left(j_{2}\right)}\right) \otimes \cdots \otimes v^{\prime}\right)\right)+\delta \circ D_{1}\left(v^{\prime} \otimes \cdots \otimes \delta^{\left(j_{1}\right)}\left(v^{\prime}\right) \otimes \cdots \otimes v^{\prime}\right) .
\end{aligned}
$$

Therefore we have

$$
\begin{aligned}
& \delta \circ D_{0}\left(v^{\prime} \otimes \cdots \otimes v^{\prime}\right)=-\sum_{k=1}^{p} k D_{1}\left(\sum_{1 \leqq j_{2}<k} v^{\prime} \otimes \cdots \otimes \delta\left(v^{\prime}\right) \otimes \cdots \otimes \delta\left(v^{\prime}\right) \otimes\right. \\
& \text { (k) } \quad\left(j_{2}\right) \\
& \left.\cdots \otimes v^{\prime}-\sum_{k<j_{2}} v^{\prime} \otimes \cdots \otimes \delta\left(v^{\prime}\right) \otimes \cdots \otimes \delta\left(v^{\prime}\right) \otimes \cdots \otimes v^{\prime}\right)+\delta \circ D_{1}\left(v^{\prime} \otimes \cdots\right. \\
& \left.\left.\otimes \delta\left(v^{\prime}\right) \otimes \cdots \otimes v^{\prime}\right)\right)+p D_{0}\left(\delta\left(v^{\prime}\right) \otimes v^{\prime} \otimes \cdots \otimes v^{\prime}\right) \\
& =-D_{1} \sum_{1 \leqq m<n \leqq p}(n-m) v^{\prime} \otimes \cdots \otimes \delta\left(\begin{array}{l}
(m) \\
v^{\prime}
\end{array}\right) \otimes \cdots \otimes \delta\left(v^{\prime}\right) \otimes \cdots \otimes v^{\prime}-\sum_{k=1}^{p} k \delta \circ D_{1} \\
& \left(v^{\prime} \otimes \cdots \otimes \delta\left(v^{\prime}\right) \otimes \cdots \otimes v^{\prime}\right)+p D_{0}\left(\delta\left(v^{\prime}\right) \otimes v^{\prime} \otimes \cdots \otimes v^{\prime}\right) \text {. }
\end{aligned}
$$

Thus we have

$$
\delta\left[D_{0}\left(v^{\prime} \otimes \cdots \otimes v^{\prime}\right)+\sum_{k=1}^{p} k D_{1}\left(v^{\prime} \otimes \cdots \otimes \delta\left(v^{\prime}\right) \otimes \cdots \otimes v^{\prime}\right)\right]
$$




$$
\begin{aligned}
= & \left.p D_{0}\left(\delta\left(v^{\prime}\right) \otimes v^{\prime} \otimes\right) \cdots \otimes v^{\prime}\right)-D_{1} \sum_{1 \leqq m<n \leqq p}(n-m) v^{\prime} \otimes \cdots \otimes \delta\left(\begin{array}{l}
(m) \\
v^{\prime}
\end{array}\right) \otimes \cdots \\
& \left.\otimes \delta\left({ }^{\prime}\right) \otimes\right) \cdots \otimes v^{\prime}
\end{aligned}
$$

By (2) we have

$$
\begin{aligned}
& D_{2} \circ \delta\left(v^{\prime} \otimes \cdots \otimes \delta\left(v^{\prime}\right) \otimes \cdots \otimes \delta\left(v^{\prime}\right) \otimes \cdots \otimes v^{\prime}\right)-\delta \circ D_{2}\left(v^{\prime} \otimes \cdots\right. \\
& \left.\otimes \delta\left(v^{\prime}\right) \otimes \cdots \otimes \delta\left({ }^{\prime}\right) \otimes \cdots \otimes v^{\prime}\right) \\
& =D_{1}\left(\sum_{j=0}^{p-n} v^{\prime} \otimes \cdots \otimes \delta\left(v^{\prime}\right) \otimes \cdots \otimes \delta\left(v^{\prime}\right) \otimes \cdots \otimes v^{\prime}-\sum_{j=1}^{n-m} v^{\prime} \otimes \cdots\right. \\
& \otimes \delta\left(v^{(j)}\right) \otimes \cdots \otimes \delta\left(v^{\prime}\right) \otimes \cdots \otimes v^{\prime}+\sum_{j=1}^{m-1} v^{\prime} \otimes \cdots \otimes \delta\left(v^{\prime}\right) \otimes \cdots \otimes \delta^{(n-m+j)}\left(v^{\prime}\right) \otimes \cdots \otimes v^{\prime} \\
& =D_{1}\left(\sum_{j=1}^{p+m-n} v^{\prime} \otimes \cdots \otimes \delta\left(v^{\prime}\right) \otimes \cdots \otimes \delta\left(v^{\prime}\right) \otimes \cdots \otimes v^{\prime}-\sum_{j=1}^{n-m} v^{\prime} \otimes \cdots \otimes \delta\left(v^{\prime}\right) \otimes\right. \\
& \cdots \stackrel{(p-n+m+j)}{\otimes} \delta\left(v^{\prime}\right) \otimes\left(\cdots \otimes v^{\prime}\right)
\end{aligned}
$$

Therefore we have

$$
\begin{aligned}
& \sum_{1 \leqq m<n \leqq p}\left[D_{2} \circ \delta\left(v^{\prime} \otimes \cdots \otimes \delta\left(\stackrel{(m)}{v^{\prime}}\right) \otimes \cdots \otimes \delta(\stackrel{n}{n}) \otimes \cdots \otimes v^{\prime}\right) \otimes \cdots \otimes v^{\prime}\right)-\delta \circ D_{2}\left(v^{\prime} \otimes\right. \\
& \left.\left.\cdots \otimes \delta\left(\stackrel{(m)}{v^{\prime}}\right) \otimes \cdots \otimes \delta\left(v^{\prime}\right) \otimes \cdots \otimes v^{\prime}\right)\right] \\
& =\sum_{1 \leqq m<n \leqq p} D_{1}^{p+}\left(\sum_{j=1}^{p+n-n} v^{\prime} \otimes \cdots \otimes \delta\left(v^{\prime}\right) \otimes \cdots \otimes \delta^{(n-m+j)}\left(v^{\prime}\right) \otimes \cdots \otimes v^{\prime}-\sum_{j=1}^{n-m} v^{\prime} \otimes\right. \\
& \left.\cdots \otimes \delta\left(\stackrel{(j)}{v^{\prime}}\right) \otimes \cdots \stackrel{(p-n+m+j)}{\otimes} \delta\left(v^{\prime}\right) \otimes \cdots \otimes v^{\prime}\right) \\
& =D_{1} \sum_{1 \leqq m<n \leqq p}[p-2(n-m)] v^{\prime} \otimes \cdots \otimes \delta\left(v^{\prime}\right) \otimes \cdots \otimes \delta\left(v^{\prime}\right) \otimes \cdots \otimes v^{\prime} \\
& =p D_{1} \sum_{1 \leqq m<n \leqq p} v^{\prime} \otimes \cdots \otimes \delta\left(v^{\prime}\right) \otimes \cdots \otimes \delta\left(v^{\prime}\right) \otimes \cdots \otimes v^{\prime}-2 D_{1} \sum_{1 \leqq m<n \leqq \phi} \\
& (n-m) v^{\prime} \otimes \cdots \otimes \delta\left(\stackrel{(m)}{v^{\prime}}\right) \otimes \cdots \otimes \delta\left(v^{\prime}\right) \otimes \cdots \otimes v^{\prime}
\end{aligned}
$$

Finally we have

$$
\begin{aligned}
& \delta\left[D_{0}\left(v^{\prime} \otimes \cdots \otimes v^{\prime}\right)+\sum_{k=1}^{p} k D_{1}\left(v^{\prime} \otimes \cdots \otimes \delta\left(v^{\prime}\right) \otimes \cdots \otimes v^{\prime}\right)\right] \\
= & p D_{0}\left(\delta\left(v^{\prime}\right) \otimes v^{\prime} \otimes \cdots \otimes v^{\prime}\right)+1 / 2\left[\sum _ { 1 \leqq m < n \leqq p } D _ { 2 } \circ \delta \left(v^{\prime} \otimes \cdots \otimes \delta\left(v^{\prime}\right) \otimes\right.\right.
\end{aligned}
$$




$$
\begin{aligned}
& \left.\cdots \otimes \delta\left(\stackrel{(n)}{v^{\prime}}\right) \otimes \cdots \otimes\right) \\
& \left.-p v^{\prime}\right)-\sum_{1 \leqq m<n \leqq p} \delta \cdot D_{2}\left(v^{\prime} \otimes \cdots \otimes \delta\left(\stackrel{(m)}{v^{\prime}}\right) \otimes \cdots \otimes \delta\left(\stackrel{(n)}{v^{\prime}}\right) \otimes \cdots \otimes v^{\prime}\right) \\
& \sum_{1 \leqq m<n \leqq p} v^{\prime} \otimes \cdots \otimes \delta\left(\stackrel{(m)}{\left.v^{\prime}\right)} \otimes \cdots \otimes \delta\left(\stackrel{(n)}{v^{\prime}}\right) \otimes \cdots \otimes v^{\prime}\right] .
\end{aligned}
$$

Obviously the $G_{h+1}$ image of the left side of (4) is zero, and the $G_{1}$ image of

$$
D_{0}\left(v^{\prime} \otimes \cdots \otimes v^{\prime}\right)+\sum_{k=1}^{p} k D_{1}\left(v^{\prime} \otimes \cdots \otimes \delta\left(v^{\prime}\right) \otimes \cdots \otimes v^{\prime}\right)
$$

coincides with $\left(G_{1}\left(v^{\prime}\right)\right)^{p}$. (4) shows that

$$
\begin{aligned}
& \left\{G_{1} 1 / p^{h+1} \delta\left[D_{0}\left(v^{\prime} \otimes \cdots \otimes v^{\prime}\right)+\sum_{k=1}^{p} k D_{1}\left(v^{\prime} \otimes \cdots \otimes \delta\left(v^{\prime}\right) \otimes \cdots \otimes v^{\prime}\right)\right]\right\} \\
& =\Delta_{p}^{h+1}(v) v^{p-1} .
\end{aligned}
$$

REMARK 2.13. (3) gives us a proof of Proposition 2.11 if $p=2$ and $r$ is even.

THEOREM 2.14. Let $x \in H^{k}\left(F, Z_{p}\right)$ be a transgressive element of even degree, and let $y \in H^{k+1}\left(B, Z_{p}\right)$ be an image of $x$ under the transgression, then

$$
d_{r} \kappa_{r}^{2}\left(y \otimes x^{p-1}\right)=0 \quad \text { for } 2 \leqq r \leqq k(p-1)
$$

and

$$
d_{s} \kappa_{s}^{2}\left(y \otimes x^{p-1}\right)=\kappa_{s}^{2}\left(-\Delta_{p}^{1} \circ \Re^{k / 2}(y) \otimes 1\right),
$$

where $s=k(p-1)+1$.

This theorem has been proved independently by T. Kudo [16] and by T. Nakamura. Partly it has been also proved by A. Borel [7]. (On the notation, see [3]]).

\section{§ 3. The operation $\Delta_{p}^{i}$ and the cohomology sequence}

Let $E / F=B$ be a fibering of a space $E$, and $A$ a principal ring. 
Then Serre [6] has proved: the sequences in the following commutative diagram

$3.1 \quad H^{\lambda+\mu-1}(F, A) \stackrel{i^{*}}{\longleftarrow} H^{\lambda+\mu-1}(E, A) \stackrel{p^{*}}{\longleftarrow} H^{\lambda+\mu-1}(B, A) \stackrel{\tau}{\longleftarrow} H^{\lambda+\mu-2}(F, A) \longleftarrow \cdots$

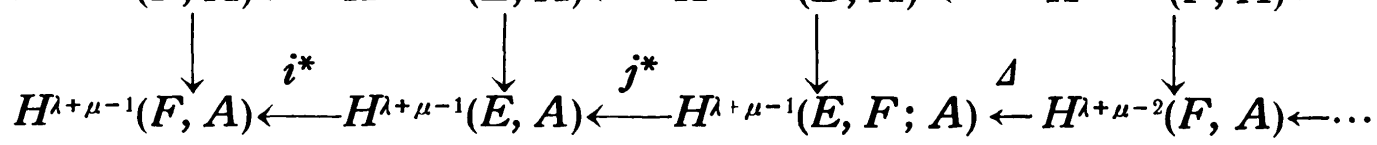
$\leftarrow H^{2}(B, A) \longleftarrow H^{1}(F, A) \leftarrow H^{1}(E, A) \leftarrow H^{1}(B, A) \longleftarrow 0$ $\leftarrow H^{2}(E, F ; A) \leftarrow H^{1}\left(\frac{\downarrow}{F}, A\right) \leftarrow H^{1}(E, A) \leftarrow H^{1}(E, F ; A) \leftarrow 0$

is exact under the conditions that the local system formed by $H^{i}(F, A)$ is trivial for each $i \geqq 0$, and $H^{i}(B, A)=0$ for

$$
0<i<\lambda \text { and } H^{i}(F, A)=0 \text { for } 0<i<\mu .
$$

Hence we can obtain some informations about the effect of $\Delta_{p}^{z}$. in $H^{*}\left(F, Z_{p}\right)$ if we know the effects of $p^{*}$ and of $\Delta_{p}^{i}$ in $H^{*}\left(E, Z_{p}\right)$ and in $H^{*}\left(B, Z_{p}\right)$, in utilizing the lower exact sequence.

The notations $i^{*}, j^{*}$ will keep the meaning in this lower sequence also in the sequel.

Now we have

THEOREM 3.2. Let $\alpha$ and $\beta$ be respectively elements of $H^{s}\left(E, Z_{p}\right)$ and of $H^{s+1}\left(E, F ; Z_{p}\right)$ such that $\delta_{r-1}(\alpha)=0$ and $\Delta_{p}^{r}(\alpha)=j^{*}(\beta) \bmod \delta_{r-1}^{\prime}$ image. Then we have

$$
\Delta \circ \Delta_{p}^{r+} \circ i^{*}(\alpha)=-\Delta_{p}^{1}(\beta) \bmod \Delta \circ \delta_{r}^{\prime} H^{s}\left(F, Z_{p^{r}}\right) .
$$

Proof. (Cf.:_diagram:(8)). 


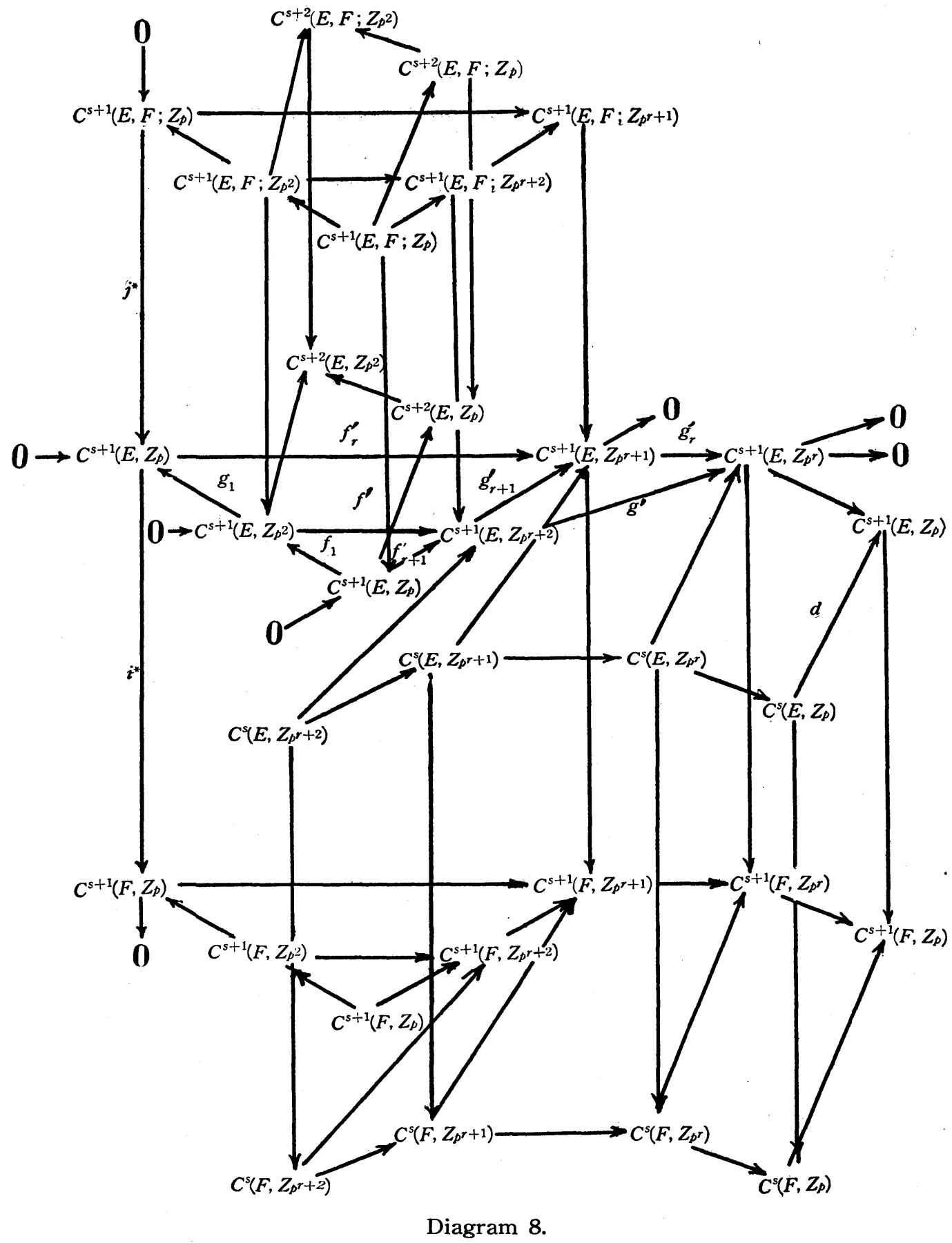

Let $a, b, c, e$ and $f$ be respectively a cocycle of $C^{s}\left(E, Z_{p}\right)$, a cocycle of $C^{s}\left(E, Z_{p^{r}}\right)$, an element of $C^{s}\left(E, Z_{p^{r+1}}\right)$, a cocycle of $C^{s+1}\left(E, F ; Z_{p}\right)$ and 
an element of $C^{s+1}\left(E, F ; Z_{p^{2}}\right)$ such that $\{a\}=\alpha, g_{r-1}(b)=a, g_{r}^{\prime}(c)=b,\{e\}=$ $\beta, g_{1}(f)=e$. By a suitable choice of $b$ and $c$, we may assume $j^{*}(e)=$ $f_{r}^{\prime-1} \circ d(c)$.

Since $j^{*}(e)=f_{r}^{\prime-1} \circ d(c), i^{*}(c)$ is a cocycle. Let $c^{\prime}$ be an element of $C^{s+1}\left(E, Z_{p^{r+2}}\right)$ whose $g_{r+1}^{\prime}$ image coincides with $c$. Since

$$
g_{r+1}^{\prime} \circ i^{*} \circ d\left(c^{\prime}\right)=g_{r+1}^{\prime} \circ d \circ i^{*}\left(c^{\prime}\right)=d \circ g_{r+1}^{\prime} \circ i^{*}\left(c^{\prime}\right)=d \circ i^{*} \circ g_{r+1}^{\prime}\left(c^{\prime}\right)=d \circ i^{*}(c)=0
$$

and $i^{*}$ is epimorphic, there exists an element $g \in C^{s+1}\left(E, Z_{p}\right)$ whose $i^{*}$ image is $f_{r+1}^{\prime-1} \circ i^{*} \circ d\left(c^{\prime}\right)$. The class $\left\{f_{r+1}^{\prime-1} \circ i^{*} \circ d\left(c^{\prime}\right)\right\} \bmod \delta_{r}^{\prime}$ image coincides with $\Delta_{p}^{r+1} \circ i^{*}(\alpha)$. Obviously we have $i^{*} \circ f_{r+1}^{\prime}(g)=i^{*} \circ d\left(c^{\prime}\right)$, that is $i^{*}\left(f_{r+1}^{\prime}(g)\right.$ $\left.-d\left(c^{\prime}\right)\right)=0$. Therefore there exists an element $h \in C^{s+1}\left(E, F ; Z_{p^{r+2}}\right)$ such that

$$
j^{*}(h)=f_{r+1}^{\prime}(g)-d\left(c^{\prime}\right)
$$

Further

because

$$
\begin{aligned}
g_{r+1}^{\prime} \circ j^{*}(h) & =g_{r+1}^{\prime}\left(f_{r+1}^{\prime}(g)-d\left(c^{\prime}\right)\right)=-g_{r+1}^{\prime} \circ d\left(c^{\prime}\right) \\
& =-d \circ g_{r+1}^{\prime}\left(c^{\prime}\right)=-d(c)=-g_{r+1}^{\prime} \circ f^{\prime} \circ j^{*}(f)
\end{aligned}
$$

$$
g_{r+1}^{\prime} \circ f^{\prime} \circ j^{*}(f)=f_{r}^{\prime} \circ g_{1} \circ j^{*}(f)=f_{r}^{\prime} \circ j^{*} \circ g_{1}(f)=f_{r}^{\prime} \circ j^{*}(e)=d(c) .
$$

This implies

$$
g_{r+1}^{\prime}\left(h+f^{\prime}(f)\right)=0 \text {. }
$$

Therefore we have $k \in C^{s+1}\left(E, F ; Z_{p}\right)$ such that

$$
f_{r+1}^{\prime}(k)=f^{\prime} \circ f_{1}(k)=h+f^{\prime}(f) \text {. }
$$

The above relations show that

$$
f^{\prime}\left(f-f_{1}(k)\right)+h=0 .
$$

Thus

$$
\begin{aligned}
f^{\prime} \circ j^{*}\left(f-f_{1}(k)\right) & =j^{*} \circ f^{\prime}\left(f-f_{1}(k)\right) \\
& =-j^{*}(h)=-\left(f_{r+1}^{\prime}(g)-d\left(c^{\prime}\right)\right) .
\end{aligned}
$$

This implies

$$
\begin{aligned}
d \circ f^{\prime} \circ j^{*}\left(f-f_{1}(k)\right) & =f^{\prime} \circ j^{*} \circ d\left(f-f_{1}(k)\right) \\
& =-d \circ f_{r+1}^{\prime}(g)=-f^{\prime} \circ f_{1} \circ d(g) .
\end{aligned}
$$

Thus 


$$
\begin{aligned}
j^{*} \circ d\left(f-f_{1}(k)\right. & =j^{*} \circ f_{1} \circ f_{1}{ }^{1} \circ d\left(f-f_{1}(k)\right) \\
& =f_{1} \circ j^{*} \circ f_{1}^{-1} \circ d\left(f-f_{1}(k)\right)=-f_{1} \circ d(g),
\end{aligned}
$$

because $f^{\prime}$ is monomorphism. In other words,

$$
j^{*} \circ f_{1}^{-1} \circ d\left(f-f_{1}(k)\right)=-d(g) .
$$

This shows that

$$
\begin{aligned}
\Delta\left\{i^{*}(g)\right\} & =\Delta\left\{f_{r+1}^{\prime-1} \circ i^{*} \circ d\left(c^{\prime}\right)\right\}=\Delta \circ \Delta_{q}^{r+1} \circ i^{*}(\alpha) \\
& =-\left\{f_{1}^{-1} \circ d\left(f-f_{1}(k)\right)\right\}=-\left\{f_{1}^{-1} \circ d(f)\right\}=-\Delta_{p}^{1}(\beta) .
\end{aligned}
$$

Q. E. D.

REMARK 3.3. We have also a corresponding theorem to Theorem 2.2 on homology groups. The same remark holds also on the following theorems in $\S 3$. (We give here only theorems on cohomology groups, as we need them solely for our purpose.)

REMARK 3.4. Theorem 3.2 gives an information on the effect of $\Delta_{p}^{r}(r=1,2, \cdots)$ on $H^{*}\left(F, Z_{p}\right)$ when we have the knowledge on the effects of $j^{*}$ on $H^{*}\left(E, F ; Z_{p}\right)$, and of $\Delta_{p}^{r}(r=1,2, \cdots)$ on $H^{*}\left(E, F ; Z_{p}\right)$ and on $H^{*}\left(E, Z_{p}\right)$. We can prove in a similar way the following proposition giving an information on the effect of $\Delta_{p}^{r}(r=1,2, \cdots)$ on $H^{*}\left(E, Z_{p}\right)$ from the knowledge on the effects of $\Delta$ on $H^{*}\left(F, Z_{p}\right)$, and of $\Delta_{p}^{r}(r=1,2, \cdots)$ on $H^{*}\left(F, Z_{p}\right)$ and $H^{*}\left(E, F ; Z_{p}\right)$. (The same remark holds also on the following theorems in. §3).

Proposition. Let $\alpha$ and $\beta$ be respectively elements of $H^{s}\left(E, F ; Z_{p}\right)$ and of $H^{s}\left(F, Z_{p}\right)$ such that $\delta_{r-1}(\alpha)=0$ and $\Delta(\beta)=\Delta_{p}^{r}(\alpha) \bmod$. $\delta_{r-1}^{\prime}$ image. Then we have

$$
i^{*} \circ \Delta_{p}^{r+1} \circ j^{*}(\alpha)=-\Delta_{\dot{p}}^{1}(\beta) \bmod i^{*} \circ \delta_{\gamma}^{\prime} H^{s}\left(E, Z_{p^{r}}\right) .
$$

REMARK 3.5. Theorem 3.3 can be also generalized in the following form :

Let $\alpha$ and $\beta$ be respectively elements of $H^{s}\left(E, Z_{p}\right)$ and of $H^{s+1}(E$, $\left.F ; Z_{p}\right)$ such that $\delta_{r-1}(\alpha)=0, \delta_{t-1}(\beta)=0$ and $\Delta_{p}^{r}(\alpha)=j^{*}(\beta) \bmod \delta_{r-1}$ image. Assume that the elements of $H^{s+1}\left(E, Z_{p^{t}}\right)$, each of which has the $g_{t-1}$ image coinciding with $\Delta_{p}^{r}(\alpha)$, are contained in the $j^{*}$ image of $H^{s-1}(E$, $\left.F ; Z_{p^{t}}\right) \bmod \delta_{r-1}^{\prime} H^{s}\left(E, Z_{p^{r}}\right)$. Then we have

$$
\Delta \circ \Delta_{p}^{r+t} \circ i^{*}(\alpha)=-\Delta_{p}^{t}(\beta) \cdot \bmod \Delta \cdot \delta_{r+t-1}^{\prime} H^{s}\left(F, Z_{p^{r+t-1}}\right) .
$$


THEOREM 3.6. Let $\alpha$ and $\beta$ be elements of $H^{s}\left(E, Z_{p}\right)$ and of $H^{s}(E$, $\left.F ; Z_{p}\right)$ such that $\delta_{r}{ }_{1}(\alpha)=0(r \geqq 2), j^{*}(\beta)=\alpha$. Then there exists an element $r \in H^{s}\left(F, Z_{p}\right)$ such that

$$
\begin{aligned}
\Delta(\gamma) & =-\Delta_{p}^{1}(\beta), \\
\Delta_{p}^{r-1}(\gamma) & =i^{*} \circ \Delta_{p}^{r}(\alpha) \bmod i^{*} \circ \delta_{r-1}^{\prime} H^{s}\left(E, Z_{p^{r-1}}\right)+\delta_{r-2}^{\prime} H^{s}\left(F, Z_{p^{r-2}}\right) .
\end{aligned}
$$

Proof. (Cf. diagram (9)).

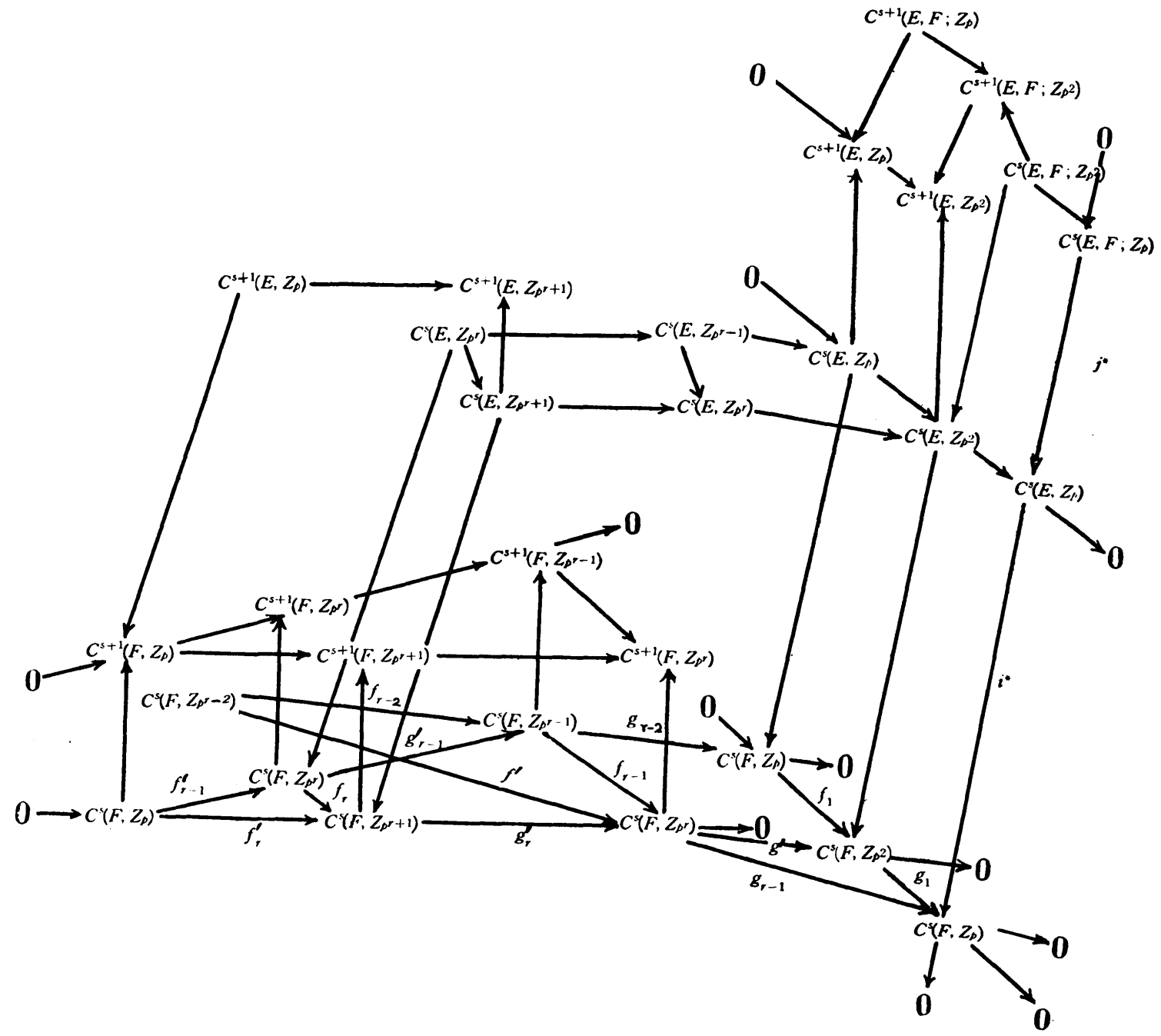

Diagram 9.

Let $a, b, c, e$ and $f$ be respectively a cocycle of $C^{s}\left(E, Z_{p}\right)$ a cocycle of $C^{s}\left(E, Z_{p^{r}}\right)$, an element of $C^{s}\left(E, Z_{p^{r-1}}\right)$, a cocycle of $C^{s}\left(E, F ; Z_{p}\right)$ and an element of $C^{s}\left(E, F ; Z_{p^{3}}\right)$ such that $\{a\}=\alpha, g_{r-1}(b)=a, g_{r}^{\prime}(c)=b,\{e\}=\beta, g_{1}(f)$ 
$=e$. Also we may assume without loss of gnerality that the $j^{*}$ image of $e$ is $a$. Then there exists an element $g \in C^{s}\left(E, Z_{p}\right)$ such that $f_{1}(g)$ $=g^{\prime}(b)-j^{*}(f)$, for we have

$$
g_{r 1}(b)=g_{1} \circ g^{\prime}(b)=a=j^{*} \circ g_{1}(f)=g_{1} \circ j^{*}(f) .
$$

Obviously $g^{\prime}(b)$ is a cocycle. Therefore

$$
\begin{aligned}
f_{1} \circ d(g) & =d \circ f_{1}(g)=-d \circ j^{*}(f)=-j^{*} \circ d(f) \\
& =-j^{*} \circ f_{1} \circ f_{1}^{-1} \circ d(f)=-f_{1} \circ j^{*} \circ f_{1}^{-1} \circ d(f) .
\end{aligned}
$$

Since $f_{1}$ is monomorphic, this implies $d(g)=-j^{*} \circ f_{1}^{-1} d(f)$. Of course, $\left\{f_{1}^{-1} \circ d(f)\right\}$ coincides with $\Delta_{p}^{1}(\beta)$. Therefore we have

$$
\Delta\left\{i^{*}(g)\right\}=-\Delta_{p}^{1}\{e\}=-\Delta_{p}^{1}(\beta) .
$$

On the other hand, we have

$$
\begin{aligned}
i^{*} \circ f_{1}(g) & =f_{1} \circ i^{*}(g)=i^{*}\left(g^{\prime}(b)-j^{*}(f)\right) \\
& =i^{*} \circ g^{\prime}(b)=g^{\prime} \circ i^{*}(b) .
\end{aligned}
$$

Since $g_{r-2}$ is epimorphic, there exists an element $a^{\prime} \in C^{s}\left(F, Z_{p^{r-1}}\right)$ such that $g_{r-2}\left(a^{\prime}\right)=i^{*}(g)$. Then we have

$$
g^{\prime} \circ f_{r-1}\left(a^{\prime}\right)=f_{1} \circ g_{r-2}\left(a^{\prime}\right)=f_{1} \circ i^{*}(g)=g^{\prime} \circ i^{*}(b) .
$$

Therefore there exists an element $b^{\prime} \in C^{s}\left(F, Z_{p^{r-2}}\right)$ such that

$$
f^{\prime}\left(b^{\prime}\right)=i^{*}(b)-f_{r-1}\left(a^{\prime}\right) \text {. }
$$

This implies

$$
f^{\prime}\left(b^{\prime}\right)+f_{r-1}\left(a^{\prime}\right)=i^{*}(b)-f_{r-1}\left(a^{\prime}\right)+f_{r-1}\left(a^{\prime}\right)=i^{*}(b) .
$$

Namely

$$
f_{r-1}\left(f_{r-2}\left(b^{\prime}\right)+a^{\prime}\right)=i^{*}(b),
$$

and

$$
g_{r-2}\left(f_{r-2}\left(b^{\prime}\right)+a^{\prime}\right)=g_{r-2}\left(a^{\prime}\right)=i^{*}(g) .
$$

Thus

$$
d \circ f_{r-1}\left(f_{r-2}\left(b^{\prime}\right)+a^{\prime}\right)=d \circ i^{*}(b)=i^{*} \circ d(b)=0 .
$$

This shows that $f_{r-2}\left(b^{\prime}\right)+a^{\prime}$ is a cocycle, for $f_{r-1}$ is monomorphic. As $g_{r-1}^{\prime}$ is epimorphic, there exists an element $c^{\prime} \in C^{s}\left(F, Z_{p^{r}}\right)$ such that

$$
g_{r-1}^{\prime}\left(c^{\prime}\right)=f_{r-2}\left(b^{\prime}\right)+a^{\prime} .
$$


And so we have

$$
\begin{aligned}
g_{r}^{\prime} \circ f_{r}\left(c^{\prime}\right) & =f_{r-1} \circ g_{r-1}^{\prime}\left(c^{\prime}\right)=f_{r-1}\left(f_{r-2}\left(b^{\prime}\right)+a^{\prime}\right) \\
& =i^{*}(b)=i^{*} \circ g_{r}^{\prime}(c)=g_{r}^{\prime} \circ i^{*}(c) .
\end{aligned}
$$

Therefore there exists an element $e^{\prime} \in C^{s}\left(F, Z_{p}\right)$ such that

$$
f_{r}^{\prime}\left(e^{\prime}\right)=i^{*}(c)-f_{r}\left(c^{\prime}\right) \text {. }
$$

This implies

$$
f_{r}^{\prime}\left(e^{\prime}\right)=f_{r} \circ f_{r-1}^{\prime}\left(e^{\prime}\right)=i^{*}(c)-f_{r}\left(c^{\prime}\right)
$$

and so

$$
f_{r}\left(f_{r-1}^{\prime}\left(e^{\prime}\right)+c^{\prime}\right)=i^{*}(c) \text {. }
$$

Further we have

$$
\begin{aligned}
& f_{r} \circ d \circ\left(f_{r-1}^{\prime}\left(e^{\prime}\right)+c^{\prime}\right)=d \circ f_{r}\left(f_{r-1}^{\prime}\left(e^{\prime}\right)+c^{\prime}\right) \\
= & d \circ i^{*}(c)=i^{*} \circ d(c)=f_{r}^{\prime} \circ f_{r}^{\prime-1} \circ i^{*} \circ d(c) \\
= & f_{r}^{\prime} \circ i^{*} \circ f_{r}^{\prime-1} \circ d(c)=f_{r} \circ f_{r-1}^{\prime} \circ i^{*} \circ f_{r}^{\prime-1} \circ d(c) .
\end{aligned}
$$

Since $f_{r}$ is monomorphic, we have

$$
d \circ\left(f_{r-1}^{\prime}\left(e^{\prime}\right)+c^{\prime}\right)=f_{r-1}^{\prime} \circ i^{*} \circ f_{r}^{\prime-1} \circ d(c) .
$$

This implies that

$$
\begin{array}{cc}
\left\{i^{*} \circ f_{r}^{\prime-1} \circ d(c)\right\} \text { coincides with } \Delta_{p}^{r-1}\left\{i^{*}(g)\right\} \bmod i^{*} \circ \delta_{r-1}^{\prime} \text { image } & \\
+\delta_{r-2}^{\prime} \text { image } & \text { Q. E. D. }
\end{array}
$$

REMARK 3.7. Theorem 3.6 can be also generalized in the following form :

Let $\alpha$ and $\beta$ be respectively elements of $H^{s}\left(E, Z_{p}\right)$ and of $H^{s}\left(E, F ; Z_{p}\right)$ such that $\delta_{r-1}(\alpha)=0, \delta_{l-1}(\beta)=0, r>t>0$ and $j^{*}(\beta)=\alpha$. Assume further that the elements of $H^{s}\left(E, Z_{p^{t}}\right)$, each of which has the $g_{t-1}$ image coinciding with $\alpha$, are contained in the $j^{*}$ image of $H^{s}\left(E, F ; Z_{p^{t}}\right)$. Then there exists an element $r \in H^{s}\left(F, Z_{p}\right)$ such that

$$
\begin{aligned}
\Delta(\gamma) & =\Delta_{p}^{t}(\beta), \\
\Delta_{p}^{r-t}(\gamma) & =-i^{*} \circ \Delta_{p}^{r}(\alpha) \bmod i^{*} \delta_{r-1}^{\prime} H^{s}\left(E, Z_{p^{r-1}}\right)+\delta_{r-t-1}^{\prime} H^{s}\left(F, Z_{p^{r-t-1}}\right) .
\end{aligned}
$$

THEOREM 3.8. Let $\alpha, \beta$ and $\gamma$ be respectively elements of $H^{s}\left(E, Z_{p}\right)$, of $H^{s+1}\left(E, F ; Z_{p}\right)$ and of $H^{s}\left(E, F ; Z_{p}\right)$ such that

$$
\Delta_{p}^{r}(\alpha)=j^{*}(\beta)(r \geqq 2) \text { and } \alpha=j^{*}(\gamma) \text {. }
$$


Then there exists an element $\varepsilon$ of $H^{s}\left(F, Z_{p}\right)$ with the following properties:

$$
\begin{aligned}
\Delta(\varepsilon) & =\Delta_{p}^{1}(r), \\
\Delta \circ \Delta_{p}^{r}(\varepsilon) & =\Delta_{p}^{1}(\beta) \quad \bmod \Delta \circ \delta_{\gamma-1}^{\prime} H^{s}\left(F, Z_{p^{r-1}}\right) .
\end{aligned}
$$

Proof is left to the reader. (Cf. diagram (10)). This theorem is a sort of combination of Theorem 3.2 and Theorem 3.6.

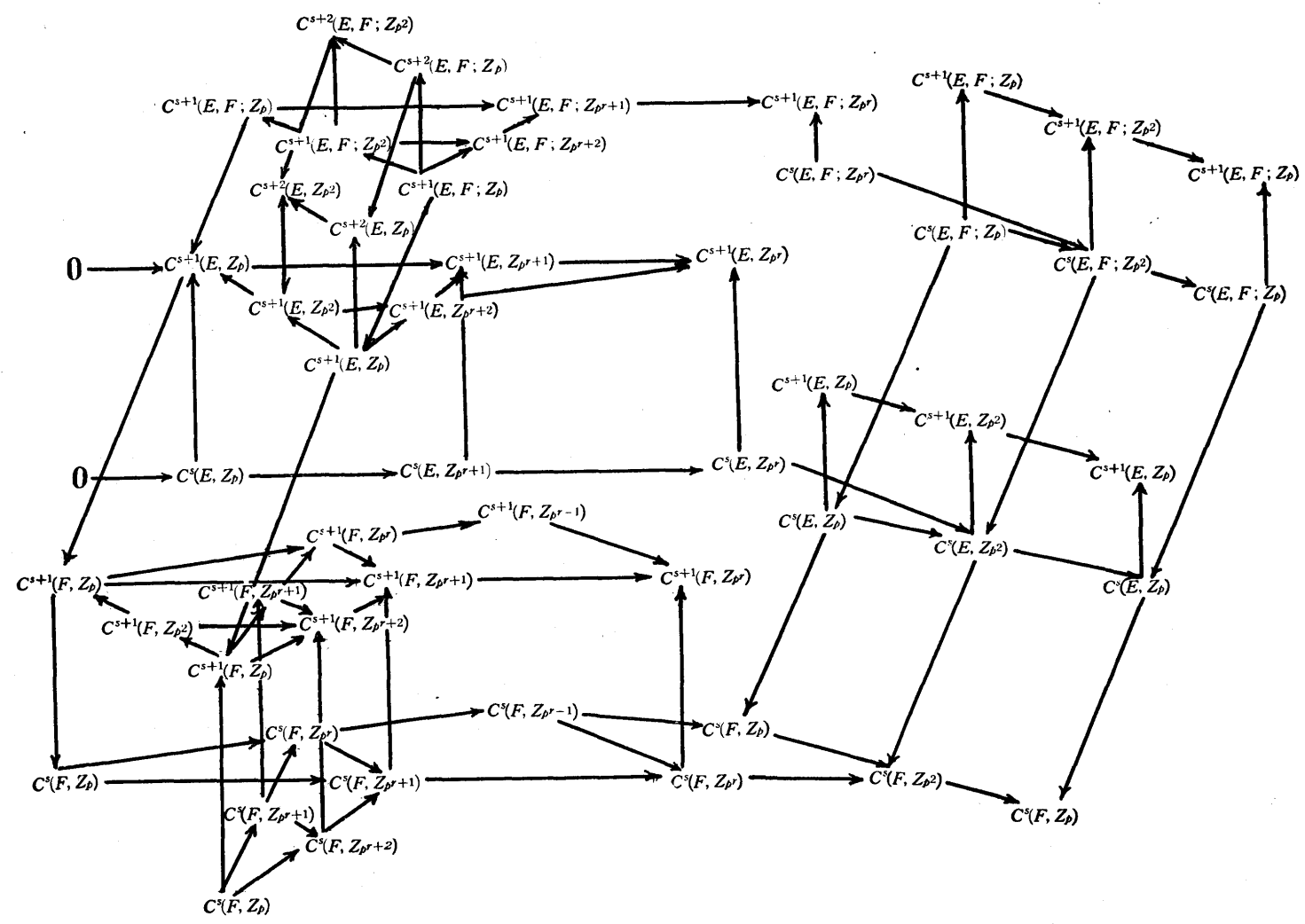

Diagram 10.

REMARK 3.9. Theorem 3.8 can be also generalized like Theorem 3.3 and Theorem 3.6. (Cf. Remark 3.5 and Remark 3.7).

THEOREM 3.10. Let $\alpha$ and $\beta$ be elements of $H^{s}\left(E, Z_{p}\right)$ and of $H^{s}\left(E, F ; Z_{p}\right)$ such that $\delta_{r-1}(\alpha)=0, \delta_{r}(\beta)=0, j^{*} \circ \delta_{r}^{\prime} H^{s}\left(E, F ; Z_{p^{r}}\right) \subset \delta_{r-1}^{\prime} H^{s}(E$, $\left.Z_{p}\right)$ and $j^{*} \circ \Delta_{p}^{r+1}(\beta)=\Delta_{p}^{r}(\alpha)$.

Moreover we assume that $j^{*}(\beta)=0$, then there exists an element $r$ of $H^{s-1}\left(F, Z_{p}\right)$ satisfying the following conditions

$$
\begin{aligned}
& \Delta(\gamma)=\beta, \\
& \Delta_{p}^{1}(\gamma)=-i^{*}(\alpha) \quad \bmod i^{*} \circ \delta_{r}^{-1}(0)\left(\subset i^{*} H^{s}\left(E, Z_{p}\right)\right)
\end{aligned}
$$




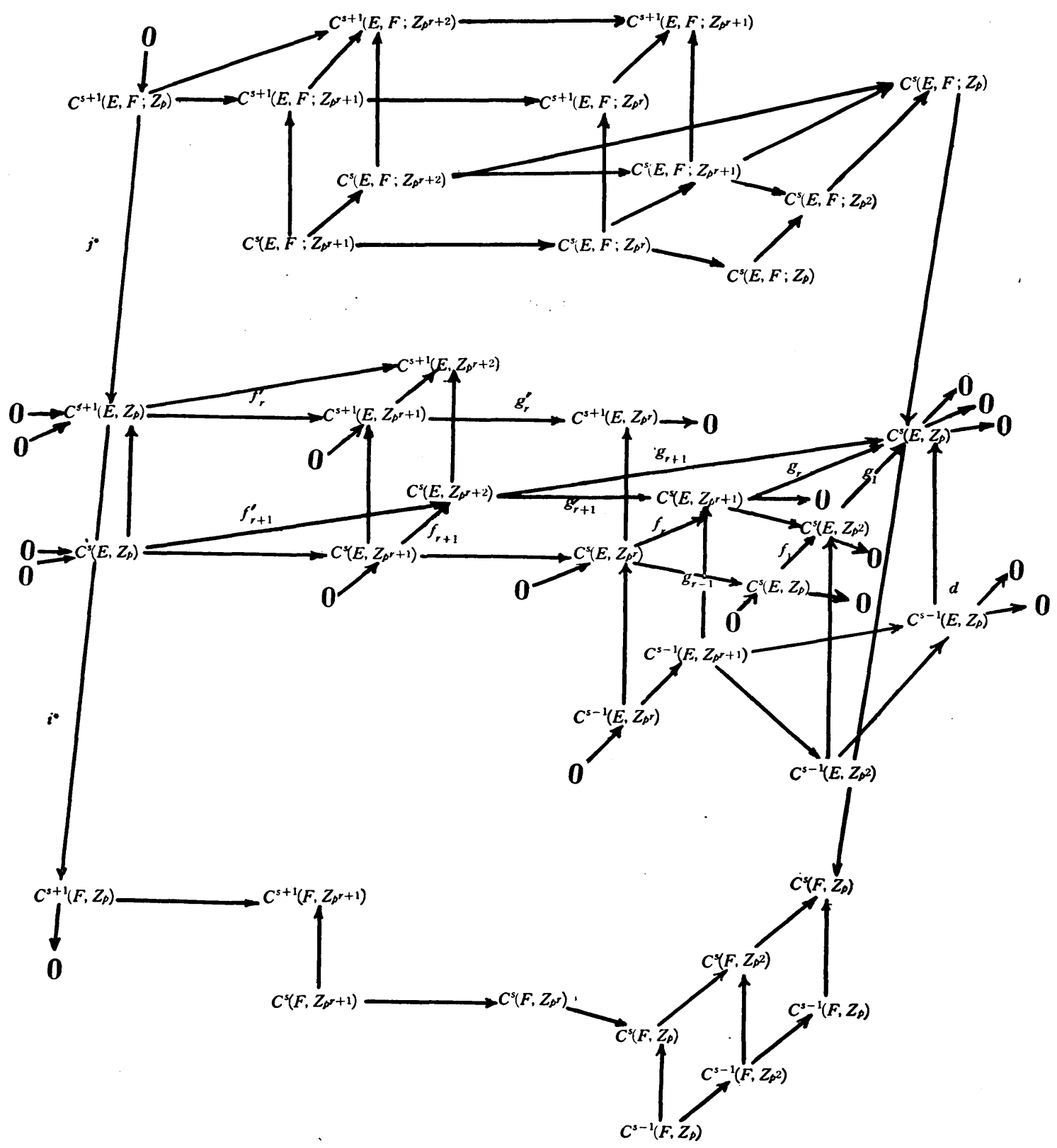

Diagram 11.

Proof. (Cf. diagram (11)). Let $a$ be a representative cocycle of $\alpha$ such that there exists a cocycle $b \in C^{s}\left(E, Z_{p^{r}}\right)$ with $g_{r-1}(b)=a$. Moreover we assume that $b$ is a $g_{r}^{\prime}$ image of $c \in C^{s}\left(E, Z_{p^{r+1}}\right)$. On the other hand, let $a^{\prime}, b^{\prime}$ and $c^{\prime}$ be respectively an element of $C^{s}\left(E, F ; Z_{p}\right)$ which represents $\beta$, a cocycle of $C^{s}\left(E, F ; Z_{p^{r+1}}\right)$ and an element of $C^{s}(E, F$; 
$Z_{p^{r+2}}$ such that $g_{r}\left(b^{\prime}\right)=a^{\prime}$ and $g_{r+1}^{\prime}\left(c^{\prime}\right)=b^{\prime}$.

Also we can assume without loss of generally

$$
j^{*} \circ f_{r+1}^{\prime-1} \circ d\left(c^{\prime}\right)=f_{r}^{\prime-1} \circ d(c),
$$

then there exists an element $e \in C^{s-1}\left(E, Z_{p^{r+2}}\right)$ such that $d_{\circ} g_{{ }_{1+1}}(e)=j^{*}\left(a^{\prime}\right)$, because $j^{*}(\beta)=0$ and $g_{r+1}$ is epimorphic. Then we have

$$
\begin{aligned}
& g_{r+1}\left(j^{*}\left(c^{\prime}\right)-f_{r+1}(c)-d(e)\right) \\
= & j^{*} \circ g_{r+1}\left(c^{\prime}\right)-d \circ g_{r+1}(e)=j^{*}\left(a^{\prime}\right)-j^{*}\left(a^{\prime}\right)=0 .
\end{aligned}
$$

Therefore there exists an element $c^{\prime} \in C^{s}\left(E, Z_{p^{r+1}}\right)$ such that

Then

$$
j^{*}\left(c^{\prime}\right)=f_{r+1}\left(c+c^{\prime \prime}\right)+d(e) .
$$

$$
j^{*} \circ d\left(c^{\prime}\right)=d \circ j^{*}\left(c^{\prime}\right)=d \circ f_{r+1}(c)+d \circ f_{r+1}\left(c^{\prime \prime}\right) .
$$

On the other hand, we have

$$
\begin{aligned}
j^{*} \circ d\left(c^{\prime}\right) & =f_{r+1}^{\prime} \circ j^{*} \circ f_{r+1}^{\prime} \circ d\left(c^{\prime}\right) \\
& =f_{r+1}^{\prime} \circ f_{r}^{\prime-1} \circ d(c)=f_{r+1} \circ d(c) .
\end{aligned}
$$

Since $f_{r+1}$ is monomorphic, this implies that $c^{\prime \prime}$ is a cocycle. Obviously $\Delta_{p}^{r}\left\{g_{r}\left(c^{\prime \prime}\right)+a\right\}$ coincides with $\Delta_{p}^{r}\{\boldsymbol{a}\}=\Delta_{p}^{r}(\alpha)$. Thus we can take $g_{r}\left(c^{\prime \prime}\right)+a$ for $a$, so we shall write a for $g_{r}\left(c^{\prime \prime}\right)+a$ from now on.

On the other hand, we have

$$
\begin{aligned}
g^{\prime} \circ g_{r+1}^{\prime} \circ j^{*}\left(c^{\prime}\right) & =g^{\prime} \circ g_{r+1}^{\prime} \circ f_{r+1}\left(c+c^{\prime \prime}\right)+g^{\prime} \circ g_{r+1}^{\prime} \circ d(e) \\
& =f_{1}(a)+g^{\prime} \circ g_{r+1}^{\prime} \circ f_{r+1}\left(c^{\prime \prime}\right)+d \circ g^{\prime} \circ g_{r+1}^{\prime}(e),
\end{aligned}
$$

and therefore

$$
\begin{aligned}
i^{*} \circ g^{\prime} \circ g_{r+1}^{\prime} \circ j^{*}\left(c^{\prime}\right) & =i^{*} \circ g^{\prime} \circ g_{r+1}^{\prime} \circ f_{r+1}\left(c+c^{\prime}\right)+i^{*} \circ d \circ g^{\prime} \circ g_{r+1}^{\prime}(e), \\
0 & =i^{*} \circ f_{1}(a)+i^{*} \circ d \circ g^{\prime} \circ g_{r+1}^{\prime}(e) .
\end{aligned}
$$

This implies

$$
i^{*}\{(a)\}=-\Delta_{p}^{1}\left\{i^{*} \circ g_{r+1}(e)\right\} .
$$

Furthermore we have

$$
g_{1} \circ d \circ g^{\prime} \circ g_{r+1}^{\prime}(e)=d \circ g_{1} \circ g^{\prime} \circ g_{r+}^{\prime}(e)=j^{*}\left(a^{\prime}\right) \text {. }
$$

This shows that

$$
\Delta\left\{i^{*} \circ g_{r+1}(e)\right\}=\left\{a^{\prime}\right\} .
$$

Q. E. D. 
REMARK 3.11. Theorem 3.10 can be also generalized in the following form :

Let $\alpha$ and $\beta$ be elements of $H^{s}\left(E, Z_{p}\right)$ and of $H^{s}\left(E, F ; Z_{p}\right)$ such that $\delta_{r-1}(\alpha)=0, \delta_{r+r^{\prime}-1}(\beta)=0\left(r^{\prime} \geqq 1\right), \delta_{r-}^{\prime} H^{s}\left(E, Z_{p^{r-1}}\right) \supset j^{*} \circ \delta_{r+r^{\prime}-1}^{\prime} H^{s}\left(E, F ; Z_{p^{r+r^{\prime}-1}}\right)$ and $j^{*} \circ \Delta_{p}^{r+r^{\prime}}(\beta)=\Delta_{p}^{r}(\alpha)$. Then there exists an element $\gamma$ of $H^{s-1}\left(F, Z_{p}\right)$ such that

$$
\begin{aligned}
\Delta(\gamma) & =\beta \\
\Delta_{p}^{r^{\prime}}(r) & =-i^{*}(\alpha) \quad \bmod \delta_{r^{\prime}-1}^{\prime} H^{s-1}\left(F, Z_{p^{r^{\prime}-1}}\right)+i^{*} \circ \delta_{r}^{-1}(0)
\end{aligned}
$$

THEOREM 3.12. Let $\alpha$ and $\beta$ be elements of $H^{s}\left(E, Z_{p}\right)$ and of $H^{s}(E$, $\left.F ; Z_{p}\right)$ such that $\left(H^{s}\left(E, Z_{p}\right) \supset\right) \delta_{r}^{-1}(0) \subset j^{*}$ image, $\delta_{r-1}(\alpha)=0, \delta_{r}(\beta)=0, j^{*}$ 。 $\delta_{r}^{\prime} H^{s}\left(E, F ; Z_{p^{r}}\right) \subset \delta_{r-1}^{\prime} H^{s}\left(E, Z_{p^{r-1}}\right), j^{*} \circ \Delta_{p}^{r+1}(\beta)=\Delta_{p}^{r}(\alpha), j^{*}(\beta)=0$ and $j^{*}(\gamma)=\alpha$, then there exists an element $\varepsilon$ of $H^{s-1}\left(F, Z_{p}\right)$ satisfying the following conditions

$$
\begin{aligned}
\Delta(\varepsilon) & =\beta \\
\Delta \circ \Delta_{p}^{2}(\varepsilon) & =\Delta_{p}^{1}(\gamma) \quad \bmod \Delta \circ \Delta_{p}^{1} H^{s}\left(F, Z_{p}\right) .
\end{aligned}
$$

PROOF. This is obtained in combining the proofs of Theorem 3.6 and of Theorem 3.10. Complete proof is left to the reader.

REMARK 3.13. In combining Remark 3.7 and Remark 3.11, we can generalize also the Theorem 3.12 .

\section{$\S 4$. A generalization of the Pontrjagin square operation and auxiliary operations}

1. A generalization of the Pontrjagin square operation.

J. H. C. Whitehead $[11,15,17]$ defined the Pontrjagin square operation

$$
P_{2}^{1}: H^{n}\left(X, Z_{2}\right) \rightarrow H^{2 n}\left(X, Z_{4}\right),
$$

with the following properties

$$
\begin{aligned}
& \text { if } n \text { is even } P_{2}^{1}(u+v)=P_{2}^{1}(u)+P_{2}^{1}(v)+f_{1}(u v), \\
& \text { if } n \text { is odd } P_{2}^{1}(u+v)=P_{2}^{1}(u)+P_{2}^{1}(v) .
\end{aligned}
$$

(2) if $n$ is even $2 P_{2}^{1}(u)=f_{1}\left(u^{2}\right)$,

if $n$ is odd $2 P_{2}^{1}(u)=0$.

We shall now generalize this operation. Denoting by $\theta$ the homo- 
morphism $H^{p n}\left(X, Z_{p^{h-1}}\right) \rightarrow H^{p n}\left(X, Z_{p^{h-1}}\right)$ induced by the injection $Z_{p^{h-1}} \rightarrow$ $Z_{p^{h+1}}$, we define an operation

$$
P_{p}^{h}: \operatorname{Ker} \delta_{h-1} \cap H^{n}\left(X, Z_{p}\right) \rightarrow H^{p n}\left(X, Z_{p^{h+1}}\right) / \theta H^{p n}\left(X, Z_{p^{h-1}}\right)
$$

in the following manner.

case $p=2$.

Let $\alpha \in \operatorname{Ker} \delta_{h-1} \cap H^{n}\left(X, Z_{2}\right)$ be represented by a cocycle $u^{\prime} \in C^{n}(X$, $\left.Z_{2}\right)$, and let $u$ be an element of $C^{n}(X, Z)$ such that $G_{1}(u)=u^{\prime}$ and $\delta(u)=2^{h} u^{\prime \prime}$. As is shown in the proof of Proposition 2.11 in $\S 2$, $G_{h+1}\left(u \smile{ }_{0} u+u \smile{ }_{1} \delta(u)\right)$ is a cocycle of $C^{2 n}\left(X, Z_{2} h+1\right)$. Now we put

$$
P_{2}^{\prime}(\alpha)=\left\{G_{h+1}\left(u \smile{ }_{0} u+u \smile_{1} \delta(u)\right)\right\} \bmod \theta H^{2 n}\left(X, Z_{2} h-1\right) .
$$

In fact $\left\{G_{h+1}\left(u \smile_{0} u+u \smile_{1} \delta(u)\right)\right\}$ is determined by $\alpha$ only, depends neither on the choices of $u^{\prime}$ and $u$, nor on the ways of performing $\smile_{0}, \smile_{1}$.

We shall verify this in the following paragraphs $1^{\circ} \sim 3^{\circ}$.

1. $\left\{\boldsymbol{G}_{h+1}\left(\boldsymbol{u} \smile_{0} \boldsymbol{u}+\boldsymbol{u} \smile_{1} \delta(u)\right)\right\}$ is independent of the ways of performing $\smile_{i}$, the choice of $u$ being already made.

Let $\smile_{i}^{0}$ and $\smile_{i}^{1}$ denote. Steenrod's $i$-products performed in two ways. Then Steenrod [9] has proved

$$
\delta\left(u_{{ }_{\mathrm{V}} 0} u\right)=u \smile{ }_{0}^{1} u-u \smile{ }_{0}^{0} u-\left[\delta(u)_{\vee_{0}} u+(-1)^{n} u_{{ } 0} \delta(u)\right]
$$

and

$$
\delta\left(u_{{ } 1} \delta(u)\right)=u \smile_{1}^{1} \delta(u)-u \smile_{1}^{0} \delta(u)-u_{{ } 0} \delta(u)+\delta(u)_{{ } 0} u-\delta(u)_{V_{1}} \delta(u) .
$$

(For the meaning of $V^{i}$ see [9]). Therefore we have

$$
\begin{gathered}
\delta\left(u_{{ } 0} u+u_{\vee_{1}} \delta(u)\right)=u \smile{ }_{0}^{1} u+u \smile{ }_{1}^{1} \delta(u)-\left(u \smile{ }_{0}^{0} u+u \smile_{1}^{0} \delta(u)\right) \\
-\left[1+(-1)^{n}\right] u_{{ } 0} 2^{h} u^{\prime \prime}-2^{2 h} u^{\prime \prime}{ }_{{ }^{1}} u^{\prime \prime}
\end{gathered}
$$

and so

$$
\begin{gathered}
G_{h+1} \circ \delta\left(u_{{ } 0} u+u_{\vee^{1}} \delta(u)\right)=G_{h+1}\left(u \smile^{1} u+u \smile_{1}^{1} \delta(u)\right) \\
-G_{h+1}\left(u \smile{ }_{0}^{0} u+u \smile_{1}^{0} \delta(u)\right)
\end{gathered}
$$

$2^{\circ} . \quad\left\{G_{h+1}\left(u \smile{ }_{0} u+u \smile_{1} \delta(u)\right)\right\}$ is independent of the choice of $\boldsymbol{u}$ (for a fixed $u^{\prime}$ ).

If $G_{1}(u)=G_{1}(v)=u^{\prime}$ and $\delta(u)=2^{h} u^{\prime \prime}, \delta(v)=2^{h} v^{\prime \prime}$, then we have $v=u$ $+2 \lambda, \lambda \in C^{n}(X, Z), \delta(\lambda)=2^{h-1} \lambda^{\prime \prime}$. Now we have 


$$
\begin{aligned}
& \left.(u+2 \lambda) \smile_{0}(u+2 \lambda)+(u+2 \lambda) \smile_{1} \delta(u)+2 \delta(\lambda)\right) \\
& \quad=u \smile_{0} u+u \smile_{1} \delta(u)+2\left(u \smile_{0} \lambda+\lambda \smile_{0} u+2 \lambda \smile_{0} \lambda+2 \lambda \smile_{1} \delta(\lambda)\right. \\
& \left.\quad+u \smile_{1} \delta(\lambda)+\lambda \smile_{1} \delta(u)\right) \\
& \quad=u \smile_{0} u+u \smile_{1} \delta(u)+2\left[-\delta\left(u \smile_{1} \lambda\right)+\left[1+(-1)^{n}\right] \lambda \smile_{0} u\right. \\
& \left.\quad+\delta(u) \smile_{1} \lambda+\left[1+(-1)^{n}\right] u \smile_{1} \delta(\lambda)+2 \lambda \smile_{0} \lambda+2 \lambda \smile_{1} \delta(\lambda)+\lambda_{1} \delta(u)\right],
\end{aligned}
$$

because the following equality holds

$$
\delta\left(u \smile_{1} \lambda\right)=-u \smile_{0} \lambda+(-1)^{n} \lambda \smile_{0} u+\delta(u) \smile_{1} \lambda+(-1)^{n} u \smile_{1} \delta(\lambda) .
$$

we have

Obviously $G_{h-1}\left(\lambda \smile_{0} u+\lambda \smile_{0} \lambda\right)$ is a cocycle in $C^{2 n}\left(X, Z_{2} h-1\right)$. Therefore

$$
\begin{aligned}
& \left\{G_{h+1}\left(v \smile_{0} v+v \smile_{1} \delta(v)\right)\right\} \\
= & \left\{G_{h+1}\left[(u+2 \lambda) \smile_{0}(u+2 \lambda)+(u+2 \lambda) \smile_{1}(\delta(u)+2 \delta(\lambda))\right]\right\} \\
= & \left\{G_{h+1}\left(u \smile_{0} u+u \smile_{1} \delta(u)\right)\right\}+\left\{\theta \cdot G _ { h - 1 } \left[\left[1+(-1)^{n}\right] / 2 \lambda \smile_{0} u\right.\right. \\
+ & \left.\left.\lambda \smile_{0} \lambda\right]\right\}=G_{h+1}\left(u \smile_{0} u+u \smile_{1} \delta(u)\right\} \bmod \theta H^{2 n}\left(X, Z_{2} h-1\right) .
\end{aligned}
$$

3०. $\left\{G_{h+1}\left(u \smile{ }_{0} u+u \smile_{1} \delta(u)\right)\right.$ is independent of the choice of $u^{\prime}$.

If $\left\{u^{\prime}\right\}=\left\{v^{\prime}\right\}=\alpha, G_{1}(u)=u^{\prime}, \delta(u)=2^{h} u^{\prime \prime}$, then we have $v^{\prime}=u^{\prime}+\delta\left(w^{\prime}\right)$, $w^{\prime} \in C^{n-1}\left(X, Z_{2}\right)$. Taking any $w \in C^{n-1}(X, Z)$ with $G_{1}(w)=w^{\prime}$ and putting $v=u+\delta(w)$, we now have $G_{1}(v)=v^{\prime}$, and further

$$
\begin{aligned}
& v \smile_{0} v+v \smile_{1} \delta(v) \\
= & (u+\delta(w)) \smile_{0}(u+\delta(w))+(u+\delta(w)) \smile_{1} \delta(u) \\
= & u \smile_{0} u+u \smile_{1} \delta(u)+u \smile_{0} \delta(w)+\delta(w) \smile_{0} u+\delta(w) \smile_{0} \delta(w) \\
+ & \delta(w) \smile_{1} \delta(u) \\
= & u \smile_{0} u+u \smile_{1} \delta(u)-\delta\left(u \smile_{1} \delta(w)\right)+\left[1+(-1)^{n}\right] \delta(w) \smile_{0} u \\
+ & \delta(w) \smile_{0} \delta(w)+\delta(u) \smile_{1} \delta(w)+\delta(w) \smile_{1} \delta(u) \\
= & u \smile_{0} u+u \smile_{1} \delta(u)-\delta\left(u \smile_{1} \delta(w)\right)+\left[1+(-1)^{n}\right] \delta\left(w \smile_{0} u\right) \\
+ & {\left[1+(-1)^{n}\right] w \smile_{0} \delta(u)+\delta\left(w \smile_{0} \delta(w)\right)-\delta\left(\delta(u) \smile_{2} \delta(w)\right), }
\end{aligned}
$$

because of the equalities:

$$
\begin{aligned}
& \delta\left(u \smile_{1} \delta(w)\right)=-u \smile_{0} \delta(w)+(-1)^{n} \delta(w) \smile_{0} u+\delta(u) \smile_{1} \delta(w), \\
& \delta\left(w \smile_{0} u\right)=\delta(w) \smile_{0} u+(-1)^{n-1} w \smile_{0} \delta(u),
\end{aligned}
$$


and

$$
\delta\left(\delta(u) \smile_{2} \delta(w)\right)=-\delta(u) \smile_{1} \delta(w)-\delta(w) \smile_{1} \delta(u) .
$$

Thus

$$
\left\{G_{h+1}\left(v \smile_{0} v+v \smile_{1} \delta(v)\right)\right\}=\left\{G_{h+1}\left(u \smile_{0} u+u \smile_{1} \delta(u)\right)\right\} .
$$

Case $\boldsymbol{p}>2$.

Let $\alpha \in \operatorname{Ker} \delta_{h-1} \cap H^{n}\left(X, Z_{p}\right)$ be represented by a cocycle $u^{\prime} \in$ $C^{n}\left(X, Z_{p}\right)$ and let $u$ be an element of $C^{n}(X, Z)$ such that

$$
G_{1}(u)=u^{\prime} \text { and } \delta(u)=p^{h} u^{\prime \prime} \text { for some } u^{\prime \prime} \in C^{n-1}(X, Z) \text {. }
$$

First let $n$ be even. As is shown in the proof of Proposition 2.13 in $\S 2$,

$$
G_{h+1}\left(D_{0}(u \otimes \cdots \otimes u)+\sum_{k=1}^{p} k D_{1}(u \otimes \cdots \otimes \delta(u) \otimes \cdots \otimes u)\right)
$$

is a cocycle of $C^{p n}\left(X, Z_{p^{n+1}}\right)$. We set

$$
\begin{gathered}
P_{p}^{h}(\alpha)=\left\{G_{h+1}\left(D_{0}(u \otimes \cdots \otimes u)+\sum_{k=1}^{p} k D_{1}(u \otimes \cdots \otimes \delta(u) \otimes \cdots \otimes u)\right)\right\} \\
\bmod \theta H^{p n}\left(X, Z_{p^{h-1}}\right)
\end{gathered}
$$

This is again determined only by $\alpha$, independently of $D_{i}$ operators and of the choices of $u^{\prime}, u$. In fact:

$$
1^{\circ} . \quad\left\{G_{h+1}\left(D_{0}(u \otimes \cdots \otimes u)+\sum_{k=1}^{p} k D_{1}(u \otimes \cdots \otimes \delta(u) \otimes \cdots \otimes u)\right)\right\}
$$

is independent of the choice of $D_{i}$.

Let $E_{i}$ be the Steenrod's notation [10], then there exists the following properties.

$$
\begin{aligned}
& E_{0}=0 \text { and } \\
& \qquad \begin{array}{l}
E_{i+1} \circ \delta+(-1)^{i} \delta \circ E_{i+1}=D_{i}-D_{i}^{\prime}-E_{i}(-1+T) \text { if } i \text { is odd, } \\
E_{i+1} \circ \delta+(-1)^{i} \delta \circ E_{i+1}=D_{i}-D_{i}^{\prime}-E_{i}\left(1+T+\cdots+T^{p-1}\right) \text { if } i \text { is even. }
\end{array}
\end{aligned}
$$

Now we have

$$
\begin{aligned}
E_{1} \circ \delta(u & \otimes \\
\quad= & E_{1} \sum_{j=1}^{p}(u \otimes u)+\delta \circ E_{1}(u \otimes \cdots \otimes u) \\
& =\left(D_{0}-D_{0}^{\prime}\right)(u \otimes \cdots(u) \otimes \cdots \otimes u)+\delta \circ E_{1}(u \otimes \cdots \otimes u)
\end{aligned}
$$


and

$$
\begin{aligned}
& E_{2} \circ \delta\left(u \otimes \cdots \otimes \delta(\stackrel{(k)}{u)} \otimes \cdots \otimes u)-\delta \circ E_{2}(u \otimes \cdots \otimes \delta(\stackrel{(k)}{u)} \otimes \cdots \otimes u)\right.
\end{aligned}
$$

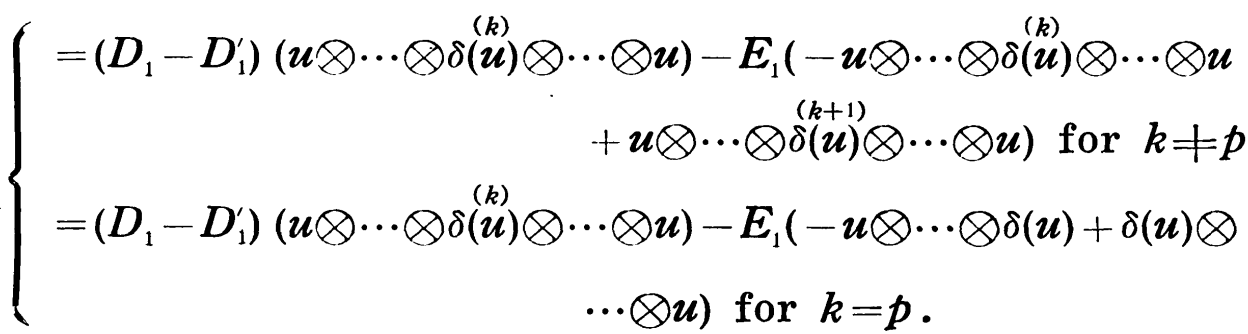

Thus we have

$$
\begin{aligned}
& E_{2} \sum_{k=1}^{p} k \delta(u \otimes \cdots \otimes \delta(u) \otimes \cdots \otimes u)-\delta \sum_{k=1}^{p} k E_{2}(u \otimes \cdots \otimes \delta(u) \otimes \cdots \otimes u) \\
& =\sum^{p} k\left(D_{1}-D_{1}^{\prime}\right)(u \otimes \cdots \otimes \delta(u) \otimes \cdots \otimes u)+E_{1} \sum^{p}(u \otimes \cdots \otimes \delta(u) \otimes \\
& \\
& \cdots \otimes u)-p E_{1}(\delta(u) \otimes u \otimes \cdots \otimes u) \\
& =\sum_{k=1}^{p} k\left(D_{1}-D_{1}^{\prime}\right)(u \otimes \cdots \otimes \delta(u) \otimes \cdots \otimes u)+\left(D_{0}-D_{0}^{\prime}\right)(u \otimes \cdots \otimes u) \\
& -\delta E_{1}(u \otimes \cdots \otimes u)-p E_{1}(\delta(u) \otimes u \otimes \cdots \otimes u) .
\end{aligned}
$$

This implies

$$
\begin{aligned}
& \left\{G _ { h + 1 } \left[D_{0}(u \otimes \cdots \otimes u)+\sum_{k=1}^{p} k D_{1}(u \otimes \cdots \otimes \delta(u) \otimes \cdots \otimes u)-\left(D_{0}^{\prime}(u \otimes \cdots\right.\right.\right. \\
& \left.\left.\left.(\otimes u)+\sum_{k=1}^{p} k D_{1}^{\prime}(u \otimes \cdots \otimes \delta(u) \otimes \cdots \otimes u)\right)\right]\right\} \\
& =\left\{G_{h+1} \circ \delta\left(\sum_{k=1}^{p} k E_{2}(u \otimes \cdots \otimes \delta(u) \otimes \cdots \otimes u)+E_{1}(u \otimes \cdots \otimes u)\right)\right\}=0,
\end{aligned}
$$

proving the stated independence.

$$
2^{\circ} . \quad\left\{G_{h+1}\left(D_{0}(u \otimes \cdots \otimes u)+\sum_{k=1}^{p} \dot{k D_{1}}(u \otimes \cdots \otimes \delta(u) \otimes \cdots \otimes u)\right)\right\}
$$

is independent of the choice of $u$ (for a fixed $u^{\prime}$ ).

If $G_{1}(u)=G_{1}(v)=u^{\prime}$ and $\delta(u)=p^{h} u^{\prime \prime}, \delta(v)=p^{h} v^{\prime \prime}$, then we have $v=u$ $+p \lambda \lambda \in C^{n}(x, Z), \delta(\lambda)=p^{h-1} \lambda^{\prime \prime}$. Now we have 


$$
\begin{aligned}
& D_{0}((\boldsymbol{u}+\boldsymbol{p} \lambda) \otimes \cdots \otimes(\boldsymbol{u}+\boldsymbol{p} \lambda))+\sum_{k=1}^{p} k D_{1}((u+p \lambda) \otimes \cdots \\
& \left.\otimes \delta\left(u^{(k)}+p \lambda\right) \otimes \cdots \otimes(u+p \lambda)\right) \\
& =D_{0}(\boldsymbol{u} \otimes \cdots \otimes u)+\sum_{k=1}^{p} k D_{1}(u \otimes \cdots \otimes \delta(u) \otimes \cdots \otimes u) \\
& +\sum_{r=1}^{p} \sum_{i_{1}<\cdots<i_{r}} p^{r} D_{0}\left(u \otimes \cdots \otimes \lambda\left(i_{1}^{\left(i_{1}\right)} \otimes \cdots \otimes \lambda \otimes \cdots \otimes u\right)+\sum_{r=1}^{p-1} \sum_{\substack{i_{1}<\cdots i_{r} \\
i \mu \neq \neq}}\right. \\
& \sum_{k=1}^{p} k p^{r} D_{1}\left(u \otimes \cdots \otimes^{\left(i_{1}\right)} \lambda \otimes \cdots \otimes \delta(u) \otimes \cdots \otimes^{(i)} \lambda \otimes \cdots \otimes u\right) \\
& +\sum_{r=1}^{p-1} \sum_{\substack{i<\cdots<i_{r} \\
i \mu \neq k}} \sum_{k=1}^{p} k p^{r+1} D_{1}\left(u \otimes \cdots \otimes \otimes^{\left(i_{1}\right)} \otimes \cdots \otimes \delta\left(\stackrel{(k)}{\lambda} \otimes \cdots \otimes^{(i r)} \lambda \otimes \cdots \otimes u\right)\right. \\
& +\sum_{k=1}^{p} k p D_{1}(u \otimes \cdots \otimes \delta(\lambda) \otimes \cdots \otimes u)
\end{aligned}
$$

A similar computation as in (3) in the proof of Proposition 2.13, $\S 2$ yields

$$
\begin{aligned}
& \sum_{i_{1}=1}^{p} D_{0}\left(u \otimes \cdots \otimes \otimes^{\left(i_{1}\right)} \lambda \otimes \cdots \otimes u\right)=-\sum_{i_{1}=1}^{p} i_{1} D_{1}\left(\sum_{\substack{j=1 \\
j \neq i_{1}}}^{p} u \otimes \cdots \otimes \lambda \otimes \cdots\right. \\
& \otimes \delta(u) \otimes \cdots \otimes u+u \otimes \cdots \otimes \delta(\lambda) \otimes \cdots \otimes u)-\sum_{i_{1}=1}^{\left(i_{1}\right)} i_{1} \delta \circ D_{1}(u \otimes \cdots \\
& \left.\otimes \lambda\left(\lambda_{1}\right) \otimes \cdots \otimes u\right)+p D_{0}(\lambda \otimes \cdots \otimes u) .
\end{aligned}
$$

Therefore

$$
\begin{aligned}
& \sum_{i_{1}=1}^{p} p D_{0}(u \otimes \cdots \otimes \lambda \otimes \cdots \otimes u)+\sum_{\substack{i_{1}=1 \\
i_{1} \neq k}}^{p} \sum_{k=1}^{p} k p D_{1}(u \otimes \cdots \otimes \delta(u) \otimes \\
& \cdots \otimes \lambda \lambda \otimes \cdots \otimes u)+\sum_{k=1}^{\left(i_{1}\right)} k p D_{1}(u \otimes \cdots \otimes \delta(\lambda) \otimes \cdots \otimes u) \\
& =p \sum_{\substack{i_{1} \\
i_{1} \neq k}}^{p} \sum_{k=1}^{p}\left(k-i_{1}\right) D_{1}(u \otimes \cdots \otimes \lambda \otimes \cdots \otimes \delta(u) \otimes \cdots \otimes u)-\sum_{i_{1}=1}^{p} \\
& i_{1} p \delta \circ D_{1}(u \otimes \cdots \otimes \lambda \otimes \cdots \otimes u)+p^{2} D_{0}(\lambda \otimes u \otimes \cdots \otimes u) .
\end{aligned}
$$

Thus we have 


$$
\begin{aligned}
& \left\{G _ { h + 1 } \left[D_{0}((u+p \lambda) \otimes \cdots \otimes(u+p \lambda))+\sum_{k=1}^{p} k D_{1}((u+p \lambda) \otimes \cdots\right.\right. \\
& \otimes(u+p \lambda) \otimes \cdots \otimes(u+p \lambda))]\} \\
& =\left\{G_{h+1}\left[D_{1}(u \otimes \cdots \otimes u)+\sum_{k=1}^{p} k D_{1}(u \otimes \cdots \otimes \delta(u) \otimes \cdots \otimes u)\right]\right\} \\
& +\theta\left\{G _ { h - 1 } \left[D_{0}(\lambda \otimes u \otimes \cdots \otimes u)+\sum_{r=2}^{p} \sum_{i_{1}<\cdots<i_{r}} p^{r-1} D_{0}(u \otimes \cdots\right.\right. \\
& \left.\left.\otimes^{\left(i_{1}\right)} \otimes \cdots \otimes^{\left({ }^{\prime}\right)}(\otimes \cdots \otimes u)\right]\right\} .
\end{aligned}
$$

Obviously

$$
\begin{aligned}
& G_{h-1}\left[D_{0}(\lambda \otimes u \otimes \cdots \otimes u)+\sum_{r=2}^{p} \sum_{i_{1}<\cdots<i_{r}} p^{r-2} D_{0}(u \otimes \cdots \otimes \lambda \otimes \cdots\right. \\
& \otimes \lambda \otimes \cdots \otimes u)]
\end{aligned}
$$

is a cocycle of $C^{p n}\left(X, Z_{p^{h-1}}\right)$. This proves our assertion.

$$
3 . \quad\left\{G_{h+1}\left[D_{0}(u \otimes \cdots \otimes u)+\sum_{k=1}^{p} k D_{1}(u \otimes \cdots \otimes \delta(u) \otimes \cdots \otimes u)\right]\right\}
$$

is independent of the choice of $u^{\prime}$.

If $\left\{u^{\prime}\right\}=\left\{v^{\prime}\right\}=\alpha, G_{1}(u)=u^{\prime}$ and $\delta(u)=p^{h} u^{\prime \prime}$, then we have $v^{\prime}=u^{\prime}+$ $\delta\left(w^{\prime}\right), w^{\prime} \in C^{n-1}\left(X, Z_{p}\right)$, and we can find $v=u+\delta(w), w \in C^{n-1}(X, Z)$ such that $G_{1}(v)=v^{\prime}$. We have immediately

$$
\begin{aligned}
& D_{0}\left((u+\delta(w)) \otimes \cdots \otimes(u+\delta(w))+\sum_{k=1}^{p} k D_{1}((u+\delta(w)) \otimes \cdots\right. \\
& \otimes \delta(\stackrel{(k)}{u}) \otimes \cdots \otimes(u+\delta(w))) \\
& =D_{0}(u \otimes \cdots \otimes u)+\sum_{k=1}^{p} k D_{1}\left(u \otimes \cdots \otimes \delta(\stackrel{(k)}{u} \otimes \cdots \otimes u)+\sum_{r=1}^{p} \sum_{i_{1}<\cdots<i_{r}}\right. \\
& D_{0}\left(u \otimes \cdots \otimes \delta(w) \otimes \cdots \otimes \delta^{\left(i_{1}\right)}(w) \otimes \cdots \otimes u\right)+\sum_{k=1}^{p} k^{p-1} \sum_{r=1}^{p-1} \sum_{\substack{i_{1} \\
i_{\mu} \neq k}} \\
& D_{1}\left(u \otimes \cdots \otimes \delta\left(\stackrel{\left(i_{1}\right)}{w}\right) \otimes \cdots \otimes \delta^{(k)}(u) \otimes \cdots \otimes \delta^{(i r)}(w) \otimes \cdots \otimes u\right)
\end{aligned}
$$
$i_{1}^{\prime}=1$.

Let $\left(i_{1}^{\prime}, \cdots, i_{r}^{\prime}\right)$ be any increasing subsequence of $(1,2, \cdots, p)$ with We denote by $\left(j_{1}^{\prime}, \cdots, j_{p-r}^{\prime}\right)$ the subsequence of $(1,2, \cdots, p)$ comple- 
mentary to $\left(i_{1}^{\prime}, \cdots, i_{r}^{\prime}\right)$. Then the following equalities can be easily verified :

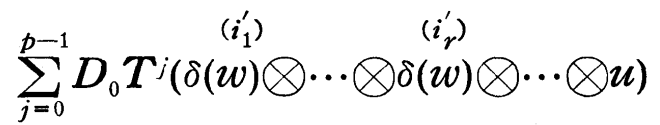

$$
\begin{aligned}
& =p D_{0}\left({ }^{\left(i_{1}^{\prime}\right)} \underset{(w)}{\left({ }^{\left(i_{r}^{\prime}\right)}\right)} \otimes \otimes \delta(w) \otimes \cdots \otimes u\right)-\sum_{j=0}^{p-1}\left[(j+1) D_{1} \circ \delta \circ T^{j}(\delta(w) \otimes\right. \\
& \left.\left.\cdots \otimes \delta(w) \otimes \cdots \otimes u)-\sum_{j=1}^{p-1}(j+1) \delta \circ D_{1} \circ T^{j}\left({ }^{\left(i_{1}^{\prime}\right)}\right) \stackrel{\left({ }^{\prime}{ }_{r}^{\prime}\right)}{(w)} \otimes \cdots \otimes \delta(w) \otimes \cdots \otimes u\right)\right], \\
& \left(i_{1}^{\prime}\right) \quad\left(i_{r}^{\prime}\right) \\
& \delta T^{j}(\delta(w) \otimes \cdots \otimes \delta(w) \otimes \cdots \otimes u) \\
& =T^{j} \sum_{k=1}^{p-1}\left(\delta \left(w_{1}^{\left(i_{1}^{\prime}\right)} \otimes \cdots \otimes \delta_{k}^{\left(i_{k}^{\prime}\right)} \otimes \cdots\left(u^{\left(i_{r}^{\prime}\right)} \otimes \cdots \otimes \delta^{\prime}(w) \otimes \cdots \otimes u\right) .\right.\right.
\end{aligned}
$$

With regard to the difference between the second summand in the right hand side of (4) and the corresponding terms in the last summand of the right hand side of (3), we have

$$
\begin{aligned}
& -\sum_{j=0}^{p-1}(j+1) D_{1} T^{j} \sum_{m=1}^{p-r}{ }^{\left({ }^{\prime}{ }_{1}^{\prime}\right)}\left(\delta(w) \otimes \cdots \otimes{ }^{\left(j_{m}^{\prime}\right)} \otimes(u) \otimes \cdots \otimes{ }^{\left(i_{r}^{\prime}\right)} \otimes(w) \otimes \cdots \otimes u\right)
\end{aligned}
$$

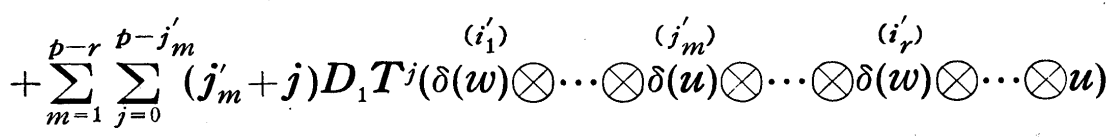

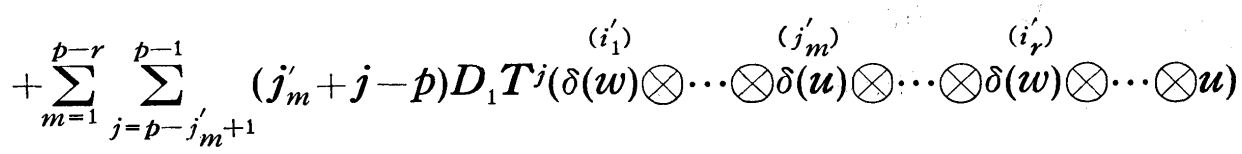

$$
\begin{aligned}
& =\sum_{m=1}^{p-r} \sum_{j=0}^{p-j_{m}^{\prime}}\left(j_{m}^{\prime}-1\right) D_{1} T^{j}\left(\delta(w) \otimes \cdots \otimes \delta^{\left(i_{1}^{\prime}\right)}(u) \otimes \cdots \otimes \delta(w) \otimes \cdots \otimes u\right)
\end{aligned}
$$

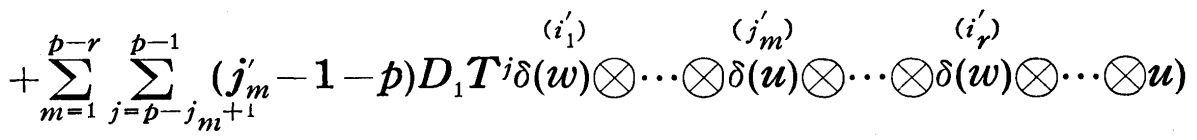

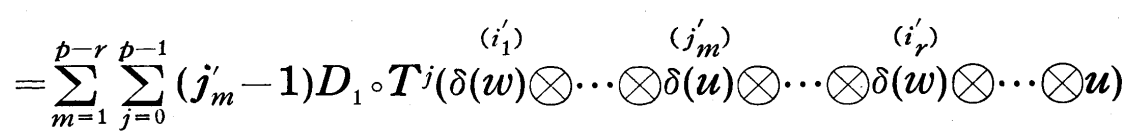

$$
\begin{aligned}
& -\sum_{m=1}^{p-r} \sum_{j=p-j_{m}^{\prime}+1}^{p-1} p D_{1} \circ T^{j}(\delta(w) \otimes \cdots \otimes \delta(u) \otimes \cdots \otimes \delta(w) \otimes \cdots \otimes u)
\end{aligned}
$$




$$
\begin{aligned}
& =\sum_{m=1}^{p-r}\left(j_{m}^{\prime}-1\right)\left[\begin{array}{c}
\left(i_{1}^{\prime}\right) \\
D_{2} \circ \delta(\delta(w)
\end{array} \otimes \cdots \otimes \delta_{m}^{\left(j_{m}^{\prime}\right)} \stackrel{\left({ }^{\prime}\right)}{\left(i_{r}\right)} \otimes \cdots \otimes \delta(w) \otimes \cdots \otimes u\right)
\end{aligned}
$$

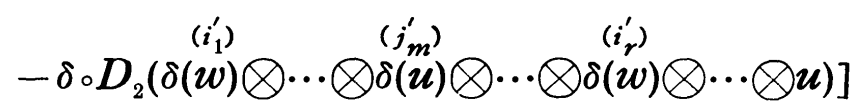

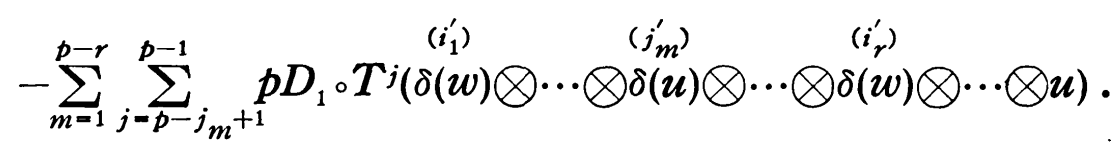

Since there exist the following equalities

$$
\begin{aligned}
& \left\{G_{h+1}\left[p D_{0}\left(\delta(w)\left(_{\left(i_{1}^{\prime}\right)}\right) \otimes \cdots \otimes \delta_{\left(i_{r}^{\prime}\right)}^{(w)} \otimes \cdots \otimes u\right]\right\}\right.
\end{aligned}
$$

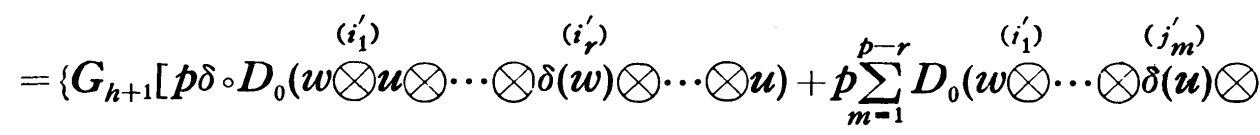

$$
\begin{aligned}
& \left(i_{r}^{\prime}\right) \\
& \cdots \otimes \delta(w) \otimes \cdots \otimes u)]\}=0,
\end{aligned}
$$

we have

$$
\begin{aligned}
& \left\{G _ { h + 1 } \left[\sum_{j=1}^{p-1} D_{0} \circ T^{i}\left({ }^{\left(i_{1}^{\prime}\right)}(w) \otimes \cdots \otimes \delta(w) \otimes \cdots \otimes u\right)+\sum_{m=1}^{p-r} \sum_{j=0}^{p-j_{m}^{\prime}}\left(j_{m}^{\prime}+j\right)\right.\right.
\end{aligned}
$$

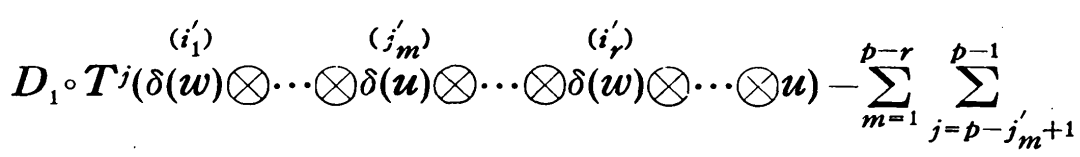

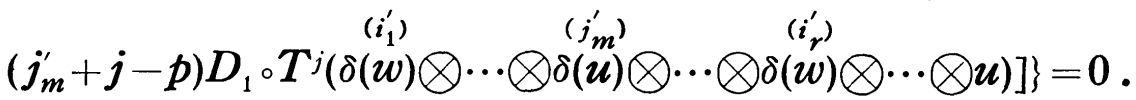

It is easily verifies that (5) is a sufficient condition for our assertion.

Next let $n$ be odd. Then we have

Q. E. D.

$$
\delta \circ D_{0}(u \otimes \cdots \otimes u)=\sum_{i=1}^{p}(-1)^{i-1} D_{0}(u \otimes \cdots \otimes \delta(u) \otimes \cdots \otimes u),
$$

and

$$
\begin{aligned}
& D_{1} \circ \delta(u \otimes \cdots \otimes \delta(\stackrel{(i)}{u}) \otimes \cdots \otimes u)+\delta \circ D_{1}(u \otimes \cdots \otimes \delta(\stackrel{(i)}{u} \otimes \cdots \otimes u) \\
& =-D_{0}(u \otimes \cdots \otimes \delta(u) \otimes \cdots \otimes u)-D_{0}(u \otimes \cdots \otimes \delta(\stackrel{(i)}{u}) \otimes \cdots \otimes u) i \leqq p-1 \\
& =D_{0}(\delta(u) \otimes \cdots \otimes u)-D_{0}(u \otimes \cdots \otimes \delta(u)) \quad i=p
\end{aligned}
$$

Therefore we have 


$$
\begin{aligned}
& \delta\left(D_{0}(u \otimes \cdots \otimes u)+\sum_{k=1}^{p} k(-1)^{k-1} D_{1}(u \otimes \cdots \otimes \delta(\stackrel{(k)}{u} \otimes \cdots \otimes u))\right. \\
& =p D_{0}\left(\delta(u) \otimes u \otimes \cdots \otimes u-\sum^{p} k(-1)^{k-1} D_{1}\left(\sum(-1)^{i-1} u \otimes \cdots \otimes \delta(\stackrel{(i)}{u} \otimes)\right.\right. \\
& \cdots \otimes \delta(\stackrel{(k)}{u}) \otimes \cdots \otimes u+\sum_{k<i \leqq p}(-1)^{i} u \otimes \cdots \otimes \delta(\stackrel{u}{u}) \otimes \cdots \otimes \delta(\stackrel{(i)}{u)} \otimes \cdots \otimes u) .
\end{aligned}
$$

This implies that

$$
\left.G_{h+1}\left[D_{0}(u \otimes \cdots \otimes u)+\sum_{k=1}^{p} k(-1)^{k-1} D_{1}(u \otimes \cdots \otimes \delta(k)) \otimes \cdots \otimes u\right)\right]
$$

is a cocycle. We set

$$
\begin{array}{r}
P_{p}^{h}(\alpha)=\left\{G_{h+1}\left[D_{0}(u \otimes \cdots \otimes u)+\sum_{k=1}^{p} k(-1)^{k-1} D_{1}(u \otimes \cdots \otimes \delta(u) \otimes \cdots \otimes u)\right]\right\} \\
\bmod \theta H^{p n}\left(X, Z_{p^{h-1}}\right) .
\end{array}
$$

This is also determined only by $\alpha$, independently of $D_{i}$ operators and of the choice of $u^{\prime}, u$. The proof is analogously performed as in the proof of the case where $n$ is even.

REMARK 1.1. In a similar way we can also define an operation

$$
P_{p}^{h}: H^{n}\left(X, Z_{p} h\right) \rightarrow H^{p n}\left(X, Z_{p^{h+1}}\right) \text {. }
$$

REMARK 1.2. If $n$ is odd, then it is easily verified that

$$
\delta_{h+1}^{\prime}\left\{G_{h+1}\left[D_{0}(u \otimes \cdots \otimes u)+\sum_{k=1}^{p} k(-1)^{k-1} D_{1}(u \otimes \cdots \otimes \delta(u) \otimes \cdots \otimes u)\right]\right\}=0 \text {. }
$$

2. Addition formula of the generalized Pontrjagin square.

In this section, we shall prove the following property of $P_{p}^{h}$ :

$$
\begin{aligned}
& P_{p}^{h}(\alpha+\beta)=P_{p}^{h}(\alpha)+P_{p}^{h}(\beta) \\
+ & f_{h}^{\prime}\left(\alpha^{p-1} \beta+\sum_{r=2}^{p-1} \frac{(p-1) \cdots(p-r+1)}{r !} \alpha^{p-r} \beta^{r}+\alpha \beta^{p-1}\right), \text { if } D(\alpha) \text { is even. } \\
& P_{p}^{h}(\alpha+\beta)=P_{p}^{h}(\alpha)+P_{p}^{h}(\beta), \text { if } D(\alpha) \text { is odd. }
\end{aligned}
$$

PROOF. of (6). Let $u$ and $v$ be cochains $\in C^{n}(X, Z)$ such that $\left\{G_{1}(u)\right\}=\alpha,\left\{G_{1}(v)\right\}=\beta$ and $\delta \circ G_{h}(u)=\delta \circ G_{h}(v)=0$. Case $p=2$. We have the following equalities

$$
\begin{aligned}
& (u+v) \smile_{0}(u+v)+(u+v) \smile_{1}(\delta(u)+\delta(v)) \\
& =u{ }_{0} u+u \smile_{1} \delta(u)+v \smile_{0} v+v \smile_{1} \delta(v)+u \smile_{0} v+v \smile_{0} u
\end{aligned}
$$




$$
\begin{aligned}
& +u \smile_{1} \delta(v)+v \smile_{1} \delta(u) \\
& =u \smile_{0} u+u \smile_{1} \delta(u)+v \smile_{0} v+v \smile_{1} \delta(v)-\delta\left(u \smile_{1} v\right)+2 u \smile_{1} \delta(v) \\
& +\left[1+(-1)^{n}\right] v \smile_{1} \delta(u)+\left[1+(-1)^{n}\right] v \smile_{0} u
\end{aligned}
$$

for

$$
\delta\left(u \smile{ }_{1} v\right)=-u \smile{ }_{0} v+(-1)^{n} v \smile{ }_{0} u+\delta(u) \smile{ }_{1} v+(-1)^{n} u \smile_{1} \delta(v) .
$$

Therefore

$$
\begin{aligned}
& G_{h+1}\left[(u+v) \smile_{0}(u+v)+(u+v) \smile_{1}(\delta(u)+\delta(v))\right] \\
& \left\{\begin{aligned}
=G_{h+1}\left(u \smile_{0} u+u \smile_{1} \delta(u)\right)+G_{h+1}\left(v \smile_{0} v+v \smile_{1} \delta(v)\right) & +f_{h}^{\prime}\left(v \smile_{0} u\right) \\
=G_{h+1}\left(u \smile_{0} u+u \smile_{1} \delta(u)\right)+G_{h+1}\left(v \smile_{0} v+v \smile_{1} \delta(v)\right) & \text { if } n \text { is even, } n \text { is odd. }
\end{aligned}\right.
\end{aligned}
$$

Case $p>2$. We give the proof only for the case $n$ is even, as the case $\boldsymbol{n}$ is odd is treated analogously.

We have the following equalities

$$
\begin{aligned}
& D_{0}((u+v) \otimes \cdots \otimes(u+v))+\sum_{k=1}^{p} k D_{1}((u+v) \otimes \cdots \otimes(\delta(u) \stackrel{(k)}{+} \delta(v)) \otimes \\
& \cdots \otimes(u+v)) \\
& =D_{0}(u \otimes \cdots \otimes u)+\sum_{k=1}^{p} k D_{1}(u \otimes \cdots \otimes \delta(u) \otimes \cdots \otimes u)+D_{0}(v \otimes \cdots \otimes v) \\
& +\sum_{k=1}^{p} k D_{1}(v \otimes \cdots \otimes \delta(v) \otimes \cdots \otimes v)+\sum_{r=1}^{p-1} \sum_{i_{1}<\cdots<i_{r}} D_{0}(u \otimes \cdots \otimes v \otimes
\end{aligned}
$$

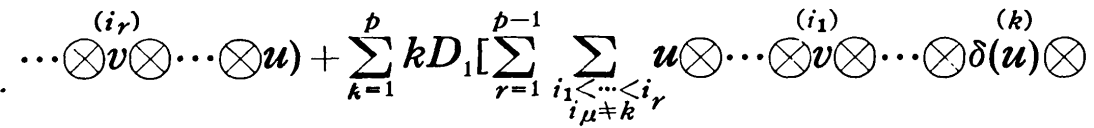

$$
\begin{aligned}
& \cdots \otimes v \otimes \cdots \otimes u+\sum_{r=1}^{p-2} \sum_{\substack{i_{1} \\
i_{\mu \neq k} \neq i_{r}}} u \otimes \cdots \otimes^{\left(i_{1}\right)} \otimes \cdots \otimes v^{(k)} \otimes(v) \otimes \cdots \otimes v^{\left(i_{r}\right)} \\
& \otimes \cdots \otimes u]+\sum_{k=1}^{p} k D_{1}(u \otimes \cdots \otimes \delta(v) \otimes \cdots \otimes u) \cdot
\end{aligned}
$$

Let $\left(i_{1}^{\prime}, \cdots, i_{r}^{\prime}\right)$ be any increasing subsequence of $(1,2, \cdots, p)$ with $i_{1}^{\prime}=1$, and $\left(j_{1}^{\prime}, \cdots, j_{p-r}^{\prime}\right)$ be the subsequence of $(1,2, \cdots, p)$ complementary to $\left(i_{1}^{\prime}, \cdots, i_{r}^{\prime}\right)$. Then the following equalities can be easily verified:

$$
\left.\sum_{j=0}^{p-1} D_{0} \circ T^{\left({ }^{\prime}{ }_{1}^{\prime}\right)}\left(v \otimes \cdots \otimes{ }^{\left(i_{r}^{\prime}\right)}\right) \cdots \otimes u\right)
$$




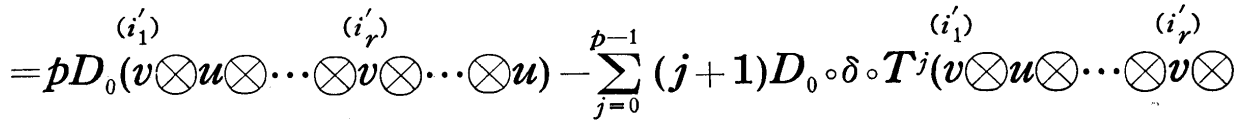

$$
\begin{aligned}
& \cdots \otimes u)-\sum_{j=0}^{p-1}(j+1) \delta \circ D_{1} \circ T^{\left(i_{1}^{\prime}\right)}\left(v \otimes \cdots \otimes v^{\left(i_{r}^{\prime}\right)} \otimes \cdots \otimes u\right), \\
& \left.\delta \circ T^{\left(i_{1}^{\prime}\right)}\left(v \otimes \cdots \otimes{ }^{\left(i_{r}^{\prime}\right)}\right) \otimes \cdots \otimes u\right)
\end{aligned}
$$

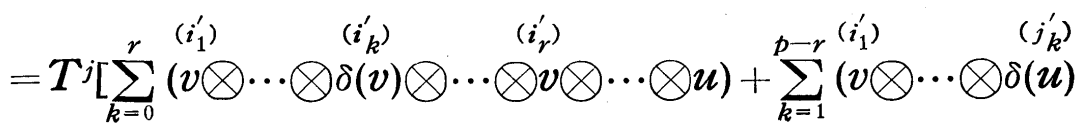

$$
\begin{aligned}
& \cdots \otimes v \otimes \cdots \otimes u)] .
\end{aligned}
$$

In virtue of the similar computation as in (4), we have

$$
\begin{aligned}
& -\sum_{j=0}^{p-1}(j+1) D_{1} T^{j}\left[\sum_{k=1}^{r} v \otimes \cdots \otimes \delta\left({ }^{\left(i_{1}^{\prime}\right)}\right) \otimes \cdots \otimes v v^{\left(i_{k}^{\prime}\right)} \otimes \cdots \otimes u+\sum_{k=1}^{p-r} v v^{\left(i_{1}^{\prime}\right)} \otimes\right.
\end{aligned}
$$

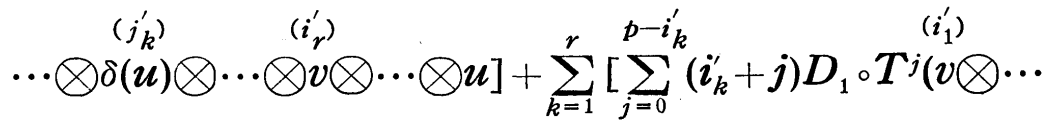

$$
\begin{aligned}
& \otimes \delta(v) \otimes \cdots\left({ }^{\left(i_{k}^{\prime}\right)} \otimes v \otimes \cdots \otimes u\right)+\sum_{j=p-i_{k}^{\prime}+1}^{p-1}\left(i_{k}^{\prime}\right)
\end{aligned}
$$

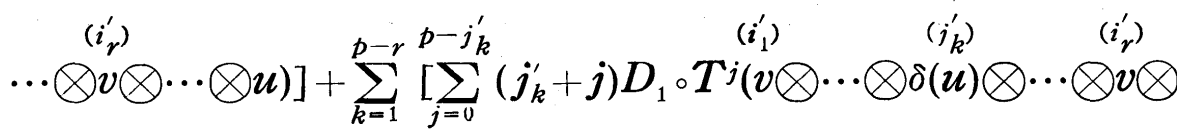

$$
\begin{aligned}
& \left.\cdots \otimes u)+\sum_{j=p-j_{k}^{\prime}+1}^{p-1}\left(j_{k}^{\prime}+j-p\right) D_{1} \circ T^{\left({ }^{\left(i_{1}^{\prime}\right)}\right.}\left(v \otimes \cdots \otimes \delta_{k}^{\left(j_{k}^{\prime}\right)} \otimes \cdots \otimes v\left({ }^{\left(i_{r}^{\prime}\right)}\right) \cdots \otimes u\right)\right] \\
& =\sum_{k=1}^{r} \sum_{j=0}^{p-1}\left(i_{k}^{\prime}-1\right) D_{1} \circ T^{\left(i_{1}^{\prime}\right)}(v \otimes \cdots \otimes \delta(v) \otimes \cdots \otimes v \otimes \cdots \otimes u) \\
& -\sum_{k=1}^{r} \sum_{j=p-i_{k}^{\prime}{ }^{+1}}^{p-1} p D_{1} \circ T^{\left(i_{1}^{\prime}\right)}\left(v \otimes \cdots \otimes \delta_{k}^{\left(i_{k}^{\prime}\right)} \otimes \cdots \otimes v^{\left(i_{r}^{\prime}\right)} \otimes \cdots \otimes u\right)
\end{aligned}
$$

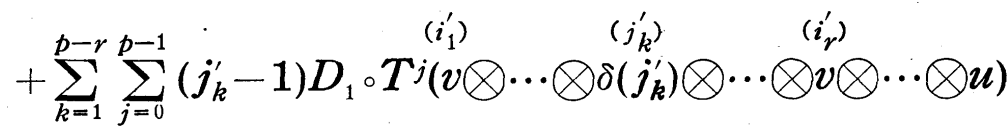

$$
\begin{aligned}
& -\sum_{k=1}^{p-r} \sum_{j=p-j_{k}^{\prime}+1}^{p-1} p D_{1} \circ T^{\left({ }^{\prime}{ }_{1}^{\prime}\right)}(v \otimes \cdots \otimes \delta(u) \otimes \cdots \otimes v \otimes \cdots \otimes u)
\end{aligned}
$$




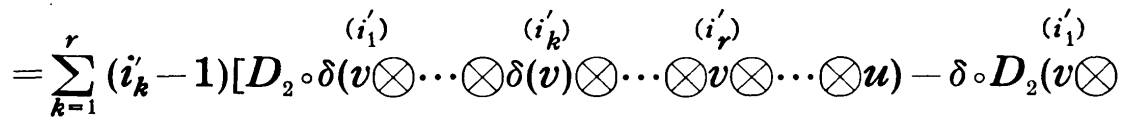

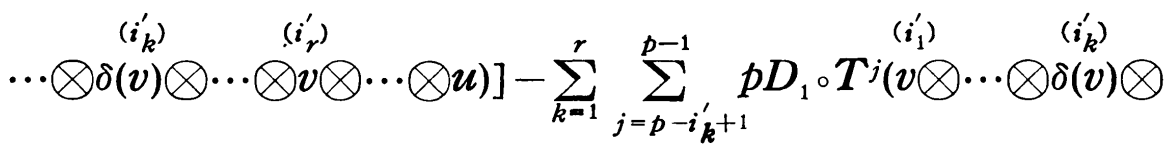

$$
\begin{aligned}
& \cdots \otimes v\left({ }_{i}^{\prime}\right)
\end{aligned}
$$

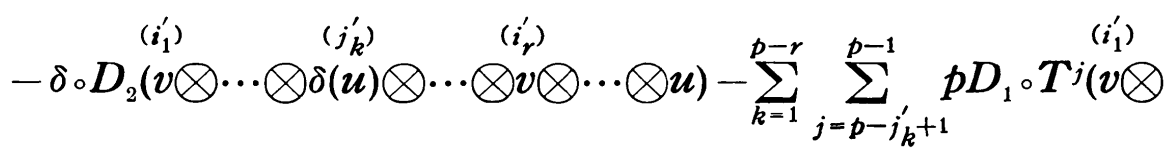

$$
\begin{aligned}
& \left.\cdots \stackrel{\left(j_{k}^{\prime}\right)}{\cdots}\left(u^{\left(i_{r}^{\prime}\right)}\right) \otimes \cdots \otimes v^{\prime} \otimes \cdots \otimes u\right)
\end{aligned}
$$

Therefore we have

(8)

$$
\begin{aligned}
& \left\{G _ { h + 1 } \left[-\sum_{j=0}^{p-1}(j+1) D_{1} \circ T^{j}\left[\sum_{k=1}^{r} v^{\left(i_{1}^{\prime}\right)} \otimes \cdots \otimes{ }^{\left(j_{k}^{\prime}\right)} \otimes(v) \otimes \cdots \otimes v^{\left(i_{r}^{\prime}\right)} \otimes \otimes \cdots \otimes u\right.\right.\right. \\
& +\sum_{k=1}^{p-r} v \stackrel{\left(i_{1}^{\prime}\right)}{v} \otimes \cdots \otimes \delta\left(u^{\left(j_{j}^{\prime}\right)} \otimes \cdots \otimes v \stackrel{\left(i_{r}^{\prime}\right)}{v} \otimes \cdots \otimes u+\sum_{k=1}^{r}\left[\sum_{j=0}^{p-i_{k}^{\prime}}\left(i_{k}^{\prime}+j\right) D_{1} \circ T^{\left(i_{1}^{\prime}\right)}(v \otimes\right.\right. \\
& \cdots \otimes \delta(v) \otimes \cdots \otimes v\left(i_{k}^{\prime}\right)
\end{aligned}
$$

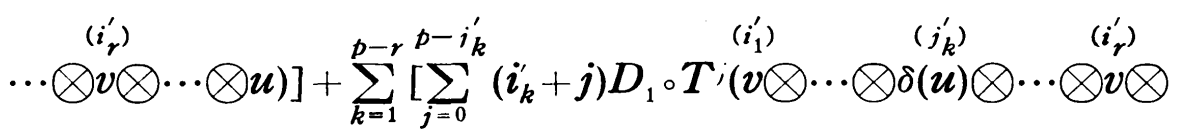

$$
\begin{aligned}
& \left.\left.\cdots \otimes u)+\sum_{j=p-j_{k}^{\prime}+1}^{p-1}\left(j_{k}^{\prime}+j-p\right) D_{1} \circ T^{\left(i_{1}^{\prime}\right)}\left(v \otimes \cdots \otimes \delta(u)\left(j_{k}^{\prime}\right) \quad \otimes \cdots \otimes v \otimes \cdots \otimes u\right]\right]\right\}=0 .
\end{aligned}
$$

It is easily verified that (8) is a sufficient condition for our assertion.

REMARK 2.1. For the operation $' P_{p}^{h}$, we have analogously

$$
\begin{gathered}
P_{p}^{h}(\alpha+\beta)=P_{p}^{h}(\alpha)+P_{p}^{h}(\beta)+f_{h}\left(\alpha^{p-1} \beta+\sum_{r=1}^{p-1}(p-1) \cdots(p-r+1)\right. \\
\left.\alpha \alpha^{p-r} \beta^{r}+\alpha \beta^{p-1}\right) .
\end{gathered}
$$

3. Auxiliary operations.

Consider the exact sequence 
(9)

$$
\cdots \rightarrow H^{p n-1}\left(X, Z_{p^{h}}\right) \rightarrow H^{p n}\left(X, Z_{p}\right) \rightarrow H^{p n}\left(X, Z_{p^{h+1}}\right) \rightarrow H^{p n}\left(X, Z_{p^{h}}\right) \rightarrow \cdots,
$$

induced by the exact sequence

$$
0 \longrightarrow Z_{p} \stackrel{f_{h}^{\prime}}{\longrightarrow} Z_{p^{h+1}} \stackrel{g_{h}^{\prime}}{\longrightarrow} Z_{p^{h}} \longrightarrow 0 \text {. }
$$

Let $\alpha$ be an element of $H^{n}\left(X, Z_{p^{h}}\right)$ such that $\alpha^{p}=0$. Then the exactness of (9) implies that there exists an element $\beta \in H^{p n}\left(X, Z_{p}\right)$ such that $f_{h}^{\prime}(\beta)=P_{p}^{h}(\alpha)$. Obviously $\beta$ is uniquely determined $\bmod \operatorname{Im} \delta_{h}^{\prime}$, and we now define

$$
\left(1 / p^{h} P_{p}^{h}\right)(\alpha)=\beta \quad \bmod \delta_{h}^{\prime} H^{p n-1}\left(X, Z_{p^{h}}\right) .
$$

Let $\alpha$ be an element of $H^{n}\left(X, Z_{p}\right)$ such that $\delta_{h-1}(\alpha)=0$ and $\alpha^{p}=0$, then by the exactness of the following sequence

$$
\cdots \rightarrow H^{p n-1}\left(X, Z_{p}\right) \rightarrow H^{p n}\left(X, Z_{p^{h}}\right) \rightarrow H^{p n}\left(X, Z_{n+1}\right) \rightarrow H^{p n}\left(X, Z_{p}\right) \rightarrow \cdots
$$

there exists an element $\gamma$ such that $f_{h}(\gamma)=P_{p}^{h}(\alpha)$.

We set

$$
\left(1 /{ }_{p} P_{p}^{h}\right)(\alpha)=r \bmod \delta_{h} H^{p n-1}\left(X, Z_{p}\right)+f_{h-1} H^{p n}\left(X, Z_{p} h-1\right) .
$$

EXAMPLE 3.1. Let $S^{n+1}(n \geqq 1)$ be an odd-dimensional sphere. Then Serre proved that $H^{*}\left(\Omega\left(S^{n+1}\right), Z\right)$ has a base $\left(\alpha_{1}, \alpha_{2}, \cdots\right)$ with $D\left(\alpha_{i}\right)=$ in $i=1,2, \cdots$ such that $\alpha_{i} \alpha_{j}=\left({ }_{i}^{i+1}\right) \alpha_{i+j}$. This shows $H^{*}\left(\Omega\left(S^{n+1}\right), Z_{p}\right)=$ $Q_{p}\left[\beta_{0}, \beta_{1}, \beta_{2}, \cdots\right], D\left(\beta_{i}\right)=p^{i}{ }_{n} i=0,1,2, \cdots$, where $Q_{p}\left[\beta_{0}, \beta_{1}, \beta_{2}, \cdots\right]$ means the factor algebra of the polynomial algebra generated by the elements $\beta_{0}, \beta_{1}, \beta_{2}, \cdots$, by the ideal generated by the elements $\beta_{0}^{p}, \beta_{1}^{p}, \beta_{2}^{p}, \cdots$. Then it is easily verified that

$$
\beta_{i+1}=1 /_{p}^{\prime} P^{1}\left(-\beta_{i}\right)\left(=1 /{ }_{p} P_{p}^{1}\left(-\beta_{i}\right)\right) \quad i=0,1,2, \cdots .
$$

REMARK 3.2. We have the following relations $(D(\alpha)$ even).

If $p>2$,

$$
\begin{aligned}
\delta_{h}^{\prime} \circ\left(1 /_{p} P_{p}^{h}\right)(\alpha)= & \alpha^{p-1} \Delta_{p}^{h}(\alpha) \\
& \bmod \delta_{h-1}^{\prime} H^{p n}\left(X, Z_{p^{h-1}}\right) .
\end{aligned}
$$

If $p=2$ and $h=1, \delta_{1}^{\prime} \circ\left(1 /{ }_{2} P_{2}^{1}\right)(\alpha)=\alpha \Delta_{2}^{1}(\alpha)+S q^{n} \Delta_{2}^{1}(\alpha)$,

if $p=2$ and $h>1, \delta_{h^{\circ}}^{\prime}\left(1 /{ }_{2} P_{2}^{h}\right)(\alpha)=\alpha \Delta_{2}^{h}(\alpha)$

$$
\bmod \delta_{h-1}^{\prime} H^{2 n}\left(X, Z_{2}{ }^{h-1}\right) \text {. }
$$

Musashi Institute of Technology and University of Tokyo. 


\section{References}

[1] J. Adem, The iteration of the Steenrod squares in algebraic topology. Proc. Nat. Acad. Sci. U.S. A. 38 (1952). pp. 720-726.

[2] J. Adem, Relations on iterated reduced powers. Proc. Nat. Acad. Sci. U.S. A. 39 (1953) pp. 636-638.

[ 3 ] A. Borel, Sur l'homologie et la cohomologie des groupes de Lie compacts connexes. Amer. Journ. of Math. 76 (1954), pp. 273-342.

[ 4 ] H. Cartan and J. P. Serre, Espaces fibrès et groupes d'homotopie I, Compt. Rend. 234 (1952) pp. 288-290.

[ 5 ] H. Cartan, Sur les groupes d'Eilenberg-MacLane, I et II. Proc. Nat. Acad. Sci. U.S. A. 40 (1954), pp. 467-471, pp. 704-707.

[6] J.P. Serre, Homologie singulière des espaces fibrès. Applications. Ann. of Math. 54 (1951), pp. 425-505.

[7] J.P. Serre, Cohomologie modulo 2 des complexes d'Eilenberg-MacLane, Comment. Math. Helv. 27 (1953) pp. 198-232.

[8] W.S. Massey, Some problem in algebraic topology and the theory of fibre bundles. Ann. of Math. 62 (1955) pp. 327-359.

[9] N.E. Steenrod, Products of cocycles and extensions of mappings. Ann. of Math. 48 (1947) pp. 290-320.

[10] N. E. Steenrod, Reduced powers of cohomology classes, Ann. of Math. 56 (1952) pp. 37-67.

[11] R. Thom, Quelques propriétés globales des variétés différentiables. Comment. Math. Helv. 28 (1954), pp. 17-86.

[12] H. Toda, Sur les groupes d'homotopie des sphères. Calcul de groupes d'homotopie des sphères, C. R. Acad. Sci. Paris, 240 (1955), pp. 42-44, 147-149.

[13] T. Yamanoshita, On the unstable homotopy groups of spheres, Proc. Jap. Nat. Acad. 31 (1955), pp. 610-611.

[14] N. Yoneda, On the homology theory of modules, Fac. Sci. Univ. Tokyo. Sec. I, Vol., 7 Part 2 (1954) pp. 193-227.

[15] J.H.C. Whitehead, On simply connected, 4-dimensional polyhedra, Comm. Math. Helv. 22 (1949), pp. 48-92.

[16] T. Kudo, A transgression theorem, Mem. Fac. Sci. Kyusyu Univ. 9 (1956)

[17] E. Thomas, A generalization of the Pontrjagin square. Proc. Nat. Acad. of Sci. U.S. A. 42 (1956) pp. 266-269. The author has seen this paper after presenting his paper to the Society. 